\title{
Probing the nature of an emergent insulator in ionic gated monolayer transition metal dichalcogenides
}

\section{Maosen Qin}

Peking University

\section{Dongdong Ding}

Peking University

Siheng Li

Peking University

Xiangyan Han

Peking University

\section{Ruirui Niu}

Peking University

\section{Dali Yin}

Peking University

Zhuangzhuang Qu

Peking University

\section{Kenji Watanabe}

National Institute for Materials Science https://orcid.org/0000-0003-3701-8119

Takashi Taniguchi

National Institute for Materials Science, Tsukuba, Ibaraki

Zhi-Min Liao

Peking University https://orcid.org/0000-0001-6361-9626

\section{Yuan Huang}

Institute of Physics, Chinese Academy of Sciences

Junren Shi

Peking University

Ji Chen

Peking University https://orcid.org/0000-0003-1603-1963

\section{Zizhao Gan}

\section{Peking University}

Jianming Lu ( $\sim$ jmlu@pku.edu.cn )

Peking University https://orcid.org/0000-0002-1558-4040 
Keywords:

Posted Date: November 10th, 2020

DOI: https://doi.org/10.21203/rs.3.rs-95219/v1

License: (c) (1) This work is licensed under a Creative Commons Attribution 4.0 International License. Read Full License 


\section{Probing the nature of an emergent insulator in ionic gated} monolayer transition metal dichalcogenides

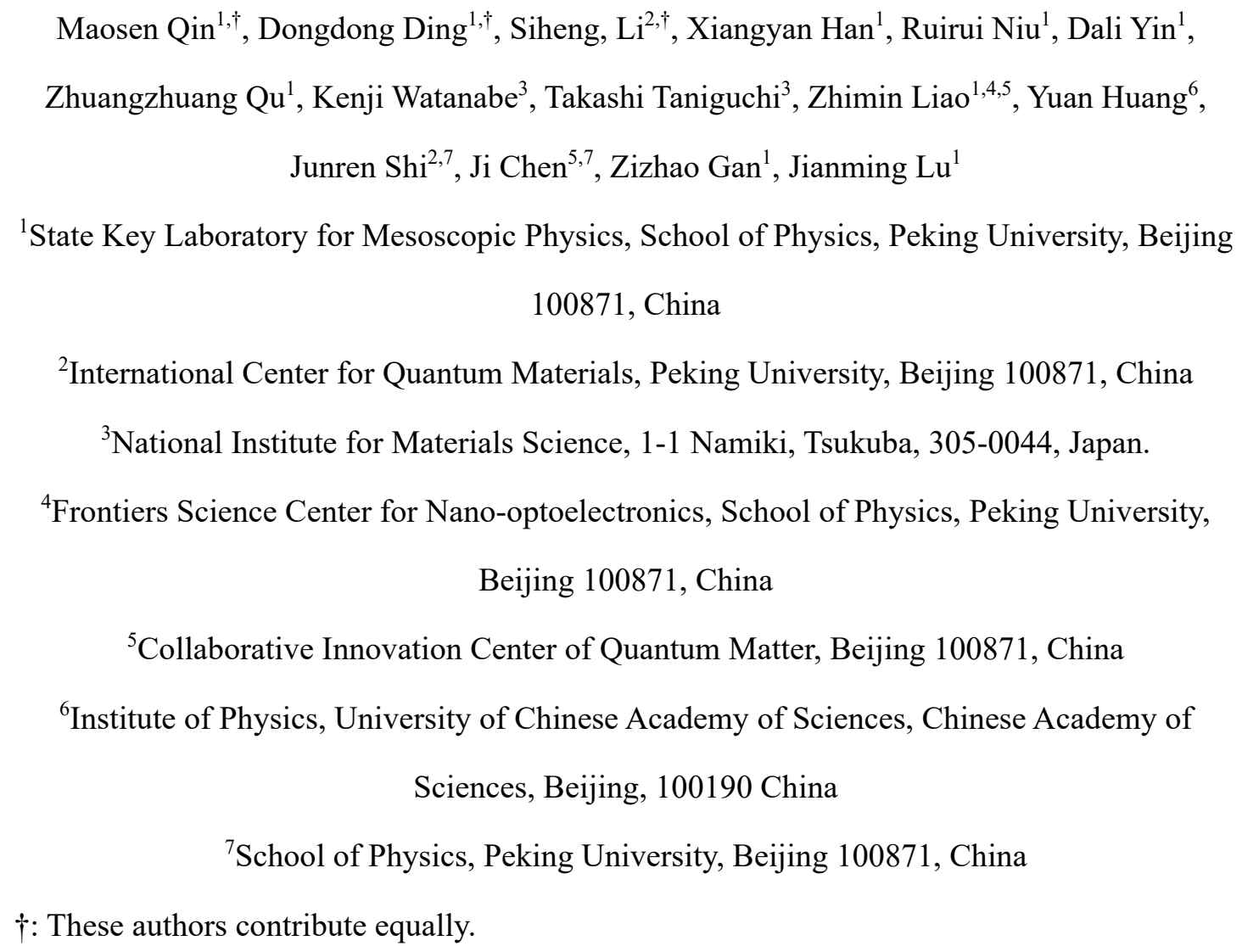

Electronic correlation in a flat band has been a longstanding interest because of emergent phenomena such as Mott insulator and superconductivity. Besides recent Moiré superlattice, transition metal dichalcogenides (TMDs) may directly, e.g. by forming a charge density wave in $1 \mathrm{~T}-\mathrm{TaS}_{2}$, reconstruct a narrow band that exhibits a correlated insulator. Here we report an emergent insulator in electron doped monolayer $\mathrm{WSe}_{2}$, a prototypical TMDs with direct bandgaps. By detailed mapping a cascade of phases "band insulator-superconductor-emergent insulator-metal", we can identify, besides the superconducting dome, a narrow miniband split from the conduction band, half filling of which coincides with the insulating state. The correlation picture is supported by a density wave that possesses an isolated flat band. Finally, through evolutionary changes within the same class of materials, multivalley population is suggested to account for enhanced superconductivity and the insulator. Our finding provides 
new opportunities to explore correlated physics within traditionally non-correlated materials.

\section{Introduction}

Whenever an insulator takes place in systems where a metal is predicted in the single particle picture, many-body physics is usually found to set in, opening an emergent energy gap at the Fermi surface. Mott insulator usually involves a narrow band composing of 'localized' orbital electrons such as $d$ electrons in copper oxide, iron pnictide, nickel oxide and $f$ electrons in heavy fermion materials ${ }^{1-4}$. With seemingly itinerant electrons, strong correlation can be introduced as well, e.g. by the confinement of Landau levels as in two-dimensional electron gas subjected to strong magnetic fields ${ }^{5}$, or by Moiré superlattice potential modulation that converts two Dirac cones into a flat band as in twisted bilayer graphene (TBG) $)^{6,7}$. Besides Mott insulator, charge density wave (CDW) could also gain an energy gap at the boundary of folded Brillouin zones in one-dimensional conductors, leading to an insulating behavior in the otherwise metallic system $^{8}$. These many-body states are possibly entangled with each other in low-dimensional systems of significant electron-electron interaction and electron-phonon coupling that are preferred by various electronic instabilities. An outstanding case in transition metal dichalcogenides is $1 \mathrm{~T}-\mathrm{TaS}_{2}$, where the so-called Davis star CDW establishes a Mott insulating phase $^{9-11}$.

Recently, two dimensional TMDs have attracted enormous attention because of exceptional physical properties such as the direct bandgap, tightly bound excitons, Berry curvature related valleytronics and Ising superconductivity ${ }^{12-21}$, many of which involve many-body effects due to quantum confinement and the reduced Coulomb screening in two-dimensional crystals ${ }^{21-23}$. Upon heavily doped with electrons, semiconducting TMDs in most cases behave like a normal Fermi liquid where single particle picture dominates the electrical transport, although negative electronic compressibility has been found to persist to carrier density in the order of $10^{13} \mathrm{~cm}^{-2}$ in $\mathrm{MoS}_{2}$ and $\mathrm{WSe}_{2}{ }^{24-27}$. In this regard, the insulating phase of a monolayer $\mathrm{WS}_{2}$ seems to be unusual as it lies on the high-density side of the Ising superconducting dome where a metal is expected ${ }^{17}$. Similar unexpected insulators were also recorded in ionic liquid gated rubrene, monolayer $\mathrm{ReS}_{2}$, silicon etc., and explained by disorder enhanced Anderson localization or 
carrier trapping induced mobility degradation ${ }^{28-35}$. Since ionic liquid was found to enhance mobility in suspended $\mathrm{MoS}_{2}$ or at least did not show increasing mobility degradation at high gate voltages $^{36-38}$, one should carefully examine the universality of localization models in these semiconducting TMDs. On the other hand, substantial doping was predicted to trigger a unique sequence of 'band insulator-superconductor-CDW' in monolayer TMDs ${ }^{39}$. Despite the proposed metallic CDW contradicts experimental observations, the involvement of CDW may shed new light on the vanishing electronic correlation (by mimicking $1 \mathrm{~T}-\mathrm{TaS} \mathrm{S}_{2}$ ) and hence the emergent insulator.

Here by taking advantage of coarse ionic liquid gating and fine dielectric gating, we are able to map in detail a cascade of electronic phases "band insulator-superconductor-emergent insulator-metal" in monolayer $\mathrm{WSe}_{2}$, a direct-bandgap semiconducting TMDs. Besides the full insulating dome indicating an intrinsic mechanism for the emergent insulator, the profile of Hall carrier density further infers that on the right side of the superconducting dome a miniband is split from the bulk conduction band, half filling of which coincides with the insulating peak. This Mott-like mechanism, as shown by density functional theory (DFT) calculations, is nicely corroborated by a $2 \sqrt{3} \times 2 \sqrt{ } 3$ charge density wave that is endowed with narrow bandwidth and heavy quasiparticles. This reconciled picture is found to be ubiquitous among doped semiconducting TMDs and the band structure evolution reveals the important role of multiplevalley population. In addition to the possible enhancement of electron-phonon coupling and hence superconductivity, the multiple Fermi surface nesting may also promote the formation of charge density wave and correlated insulator, thus providing a systematic view of superconductivity and emergent insulators in gated monolayer TMDs.

\section{Results}

Tungsten diselenide ( $\mathrm{WSe}_{2}$ ) has a trigonal prismatic crystal structure with $\mathrm{Se}$ and $\mathrm{W}$ arranged to a sandwich structure by covalent bonds in a sequence of Se-W-Se (Fig. 1a). Similar to the most intensively studied $\mathrm{MoS}_{2}, \mathrm{WSe}$ features a significant direct bandgap $(\sim 1.6 \mathrm{eV})$, a pair of time reversal symmetry related valleys at the hexagonal Brillouin zone corners, and a more 
pronounced spin splitting in valleys of both valence and conduction bands ${ }^{40}$. In the electric transport, the very attractive property may be the ultrahigh hole mobility, allowing for the observation of many interesting quantum phenomena at low temperatures ${ }^{41,42}$.

\section{Observation of an intrinsic insulator}

Here the monolayer $\mathrm{WSe}_{2}$ flake was mechanically exfoliated from bulk crystal and made into a Hall-bar device (See details in Methods, section 1). As shown in Fig. 1a, the finalized field effect transistor has a dual-gate configuration: ionic liquid as a coarse top gate $V_{\mathrm{TG}}$ and a fine dielectric capacitor as the back gate $V_{\mathrm{BG}}$. The transistor exhibits characteristic ambipolar behaviors, as shown by the conductivity of a typical sample (Fig. 1b). Although electron and hole sides are both substantially doped, reaching the order of $10^{14} \mathrm{~cm}^{-2}$, the conductivity profiles are distinct. As denoted in Fig. 1c-d, on the hole side only $K / K^{\prime}$ valleys are filled, whereas both $K$ and $Q$ valleys are occupied on the electron side because of the small energy difference between the two valleys ${ }^{40,43-47}$. The strong intervalley scattering results in a remarkable kink in conductivity, consistent with the interband scattering in multilayer graphene and previous reports for $\mathrm{MoS}_{2}, \mathrm{MoSe}_{2}$ and $\mathrm{WSe}_{2}{ }^{48-50}$. This feature is expected to depend mildly on temperature in theory, since density of states at valley minima would not be severely broadened by thermal effects ${ }^{51}$. However, as shown by the color coded resistivity (Fig. 1e and Fig. S1) as a function of temperature and effective back gate $V_{\text {eff }}$ (see derivation of $V_{\text {eff }}$ in Methods, section 2 and Fig. S2), when electron doped $\mathrm{WSe}_{2}$ was cooled down, in addition to the superconducting dome (blue color, denoted by SC) the most striking feature is an insulating dome (red color, denoted by emergent insulators/EI). Obviously, it is unreasonable to take EI as a direct development of the kink in conductivity that originates from intervalley scattering.

The insulating phase at high gate voltages of ionic liquid transistor is sometimes termed as reentrant insulator because of the band insulator without doping (denoted as BI in Fig. 1e). It has been widely observed in silicon, rubrene and monolayer TMDs, and explained by disorder induced localization ${ }^{17,28,29,32-35}$. The observation that is unique to this phase diagram, is the transition back to a metal at the highest gate voltage. Following the Anderson localization 
model of ionic liquid transistors ${ }^{28}$, cations and induced electrons in samples have a one-to-one correspondence, so a higher gate voltage (i.e. more electrons and cations) means a more disordered potential landscape. A natural expectation is, once the sample enters a disordered insulator it would stay inside the phase for higher gate voltages, contrasting the above observation in $\mathrm{WSe}_{2}$.

The challenge to the above localization model can also be seen from the effect of the dielectric gate that is supposed to bring in much less disorder than ionic liquid. On the left border of the insulating dome $\left(V_{\text {eff }} \sim 1000 \mathrm{~V}\right)$, we are able to use positive back gates to obtain a more insulating state while the ionic liquid gate voltage (and the cation distribution at the sample surface) is fixed. This is better illustrated in Fig. S4 and S5, where thin boron nitride and graphite flakes form an ideally clean capacitor ${ }^{52,53}$. Thanks to the powerful carrier control capability, the superconducting state can be directly tuned into the emergent insulator by a positive gate voltage, i.e. by putting more electrons into the system. As such, the density driven emergent insulator is not likely to result from pure disorder effects.

\section{Identification of a split narrow band}

To gain more insight, we characterize the temperature dependent resistivity and Hall carrier density in details. The behavior can be well described by Efros-Shklovskii-variable range hopping ${ }^{54}$ in the broad transition region from the emergent insulator to metal on both sides (Fig. 2a-b, and Methods, section 4), and the thermal activation model could be used to fit the most insulating states (Fig. 2c). The fitting parameters are summarized in Fig. 2d, in which localization length and the energy gap are depicted to left and right axes, respectively. The energy gap is as high as $93 \mathrm{meV}$, much larger than the energy scale $(\sim 30 \mathrm{meV})$ derived for monolayer $\mathrm{WS}_{2}{ }^{17}$. The reason is obvious: due to the limited gating capability, the insulating state of $\mathrm{WS}_{2}$ in the previous report has not yet reached its insulating dome peak. But in $\mathrm{WSe}_{2}$, it is sufficient to access the whole insulating phase. Identification of the difference between these two similar materials may be the key to understanding the emergent insulator. We will return to this point at the end of the manuscript. 
The profile of Hall carrier density across the cascade of phases would give more information (Fig. 2e). As the dielectric back gate is used to evaluate the gating effect of ionic liquid, we do not need to incorporate quantum capacitance of monolayer TMDs since the latter is roughly three orders larger in magnitude. At the initial stage of filling the conduction band, the effective gate capacitance denoted by the black dotted line is $C_{\mathrm{eff}}=4.5 \mathrm{nF} / \mathrm{cm}^{2}$, roughly $1 / 3$ of the standard model (a parallel plate capacitor with $285 \mathrm{~nm} \mathrm{SiO}_{2}$ ). This reduction of capacitance in the lowdensity regime is quite robust and has been repeatedly found in devices made of exfoliated and chemically grown $\mathrm{WS}_{2}$ (Methods, section 3 and Fig. S3) ${ }^{17}$. The exact reason is beyond the scope of this manuscript, but it may relate with trapped states below the conduction band edge. Upon passing this region, the fitted capacitance (blue dotted line) restores to be consistent with theoretical expectations. The only exception is that, when the emergent insulating state rises gradually, the Hall carrier density begins to deviate from theory and eventually decreases with increasing effective gate voltages (denoted by open symbols). As inferred from Fig. 2d, at this stage electrons are susceptible to localization but Hall measurements can only account for mobile carriers, thus the Hall carrier density cannot be directly translated to the whole electrons injected into the sample. Instead, it is more reasonable to trace the total number by following the established capacitor model. The extrapolated carrier density for the insulating peak is around $7.3 \times 10^{13} \mathrm{~cm}^{-2}$. An alternative way is to conduct Hall measurements at high temperature when the insulator is quenched. Thanks to the significant dip in the conductivity curve on the electron side (Fig. 1b), we can locate the carrier density for the insulator as $7.1 \times 10^{13} \mathrm{~cm}^{-2}$ and denote it with a solid circle (blue) in Fig. 2e. The magnitude is consistent with previous reports for the carrier density around the conductance $\mathrm{dip}^{49}$, and more importantly, it follows closely the fitting line (dotted line in blue). The good agreement between calculation and experiments, The most intriguing observation in Fig. $2 \mathrm{e}$ is the carrier profile on the right side of the insulating phase, which seems to accumulate electrons in an empty band. Before we discuss its implication, we'd like to evaluate the magnitude of "lost" electrons. Following the capacitor model, we take 
$V_{\text {eff }} \sim 2260 \mathrm{~V}$ at the intercept to the horizontal axis, and obtain $1.5 \times 10^{14} \mathrm{~cm}^{-2}$. The quantity almost doubles the carrier density at the insulating peak $\left(\sim 7.1 \times 10^{13} \mathrm{~cm}^{-2}\right)$, which immediately suggests a Mott-like mechanism in a miniband (Fig. 2g). It's interesting to compare the proposed miniband to the narrow band in monolayer $\mathrm{ReS}_{2}$, both of which are split from the conduction band of a large bandwidth. Naively, one would suppose that the disordered potential fluctuation may exceed the bandwidth and cause an insulator, just in the same way with that in $\mathrm{ReS}_{2}$. Here we emphasize that the underlying mechanism must be different, because in Anderson localization model of $\mathrm{ReS}_{2}$ it is more and more insulating at higher $V_{\text {eff }}$ whereas the states with higher $V_{\text {eff }}$ than the insulating peak in $\mathrm{WSe}_{2}$ tend to be more metallic. Once the miniband is fully filled (by the "lost" electrons), a new band at higher energies starts to be populated (Fig. 2h), and the profile of Hall carrier density is again similar to that in the initial doping stage (Fig. 2f). Overall, the above scenario can well explain our experiments, but it poses stringent requirements for the miniband: to accommodate electrons with strong correlation $\sim 1.5 \times 10^{14} \mathrm{~cm}^{-2}$.

\section{Charge density wave with a flat band}

A miniband could be formed by Moiré superlattice in $\mathrm{TBG}^{6,7}$, or more conventionally by a charge density wave in $1 \mathrm{~T}-\mathrm{MX}_{2}(\mathrm{M}=\mathrm{Ta}, \mathrm{Nb} ; \mathrm{X}=\mathrm{S}, \mathrm{Se})^{9-11,55-57}$, well-known group VB-TMDs. Notably, in semiconducting $\operatorname{MoS}_{2}$ there were already theoretical works proposing a sequence of "band insulator-superconductivity-CDW" in phase diagrams, though the CDW still has a large Fermi surface (i.e. metallic) ${ }^{39}$. Here DFT calculations were carried out (see Methods, section 7) for multiple structural instabilities with appropriate doping. Besides the previously established $2 \times 1$ CDW (denoted as $M$-CDW), we found a novel $2 \sqrt{3} \times 2 \sqrt{3}$ CDW (denoted as $Q$ CDW) whose partial charge density contour could be found in Fig. S8. Schematic Brillouin zones and CDW wavevectors are shown in Fig. 3a-b and calculated band structures are in Fig. $3 \mathrm{c}-\mathrm{d}$ for $Q$ - and $M$-CDW, respectively. The striking feature of $Q$-CDW is the emerging flat band (Fig. 3c), which is different from that either in undistorted (Fig. S6) or M-CDW phases (Fig. 3d). The bandwidth of the flat band is as narrow as $100 \mathrm{meV}$, and the effective mass as large as $20 m_{0}$, probably invoking strong electron-electron interactions that have not been observed in 
such gated semiconductors. Significantly, when the doping exceeds 0.04 e/u.c. (electron per unit cell) $\sim 4 \times 10^{13} \mathrm{~cm}^{-2}$, the $Q$-CDW becomes energetically more stable than the $M$-CDW, thus the narrow band would dominate electric transport properties in high density regimes (Fig. 3e).

The emergence of a flat band provides possibilities for insulating behaviors observed experimentally. An extended discussion of the narrow band physics is beyond the scope of this study, but it is worth mentioning a few simple scenarios. For the full filling of the narrow band, carrier densities need to reach $2 / 12$ e/u.c. $\left(\sim 1.67 \times 10^{14} \mathrm{~cm}^{-2}\right)$, so at the half filling, i.e. $1 / 12$ e/u.c. $\left(\sim 8.3 \times 10^{13} \mathrm{~cm}^{-2}\right)$ the system may turn into a Mott insulator. When slightly away from the half filling, Wigner-crystal like electronic solids may form ${ }^{58}$. For instance, if we take the dielectric constant as $7.4^{59}$ and carrier density $\sim 3 \times 10^{13} \mathrm{~cm}^{-2}$, the Wigner-Seitz radius $r_{\mathrm{s}}$, standing for the ratio between Coulomb potential and kinetic energy, reaches 51 that well exceeds the threshold of $29^{60}$. Considering cations and inevitable defects in our samples, the ideal Wigner crystal is probably replaced by an electronic solid made of interacting electrons stabilized by the local charge disorder.

It's important to stress that the flat band can accommodate electrons of $1.67 \times 10^{14} \mathrm{~cm}^{-2}$ and heavy effective mass, which fulfills the strict conditions set by experiments. However, we note that the flat band can only be stabilized by doping rates above 0.15 e/u.c. $\left(1.5 \times 10^{14} \mathrm{~cm}^{-2}\right)$, which is larger than the density where the insulator starts to be observable in experiments. The discrepancy may be due to the well-known limitation of DFT in the quantitative description of strong electronic correlations; another obvious omission is the energy gain by forming electronic solids compared to Fermi liquid, which would stabilize the Wigner-like crystal at a lower carrier density than DFT calculations.

\section{Evolution of superconductivity and the emerging insulator}

The story of the emergent insulator in gated $\mathrm{WSe}_{2}$ should not be unique, as the insulator or its preliminary sign at high gate voltages could also be found in monolayer $\mathrm{WS}_{2}$ and $\mathrm{MoS}_{2}$ that have the same crystal structure and qualitatively similar band structure. As noted earlier, the 
quantitative difference between the three would be the key to understanding the emergent insulator. In the following, we present a systematic investigation.

Compared to $\mathrm{WSe}_{2}$ (Fig. $4 \mathrm{a}$ ), $\mathrm{WS}_{2}$ possesses an almost the same superconducting dome but a less resistive insulator (Fig. 4b). As for $\mathrm{MoS}_{2}$, only a conductivity dome was clearly identified at temperatures above $2 \mathrm{~K}$ (Fig. $4 \mathrm{c}$ ). As $T_{\mathrm{c}}$ was found to be below $1.5 \mathrm{~K}$ that is beyond the measurement range, we believe a much smaller superconducting dome exists beneath the conductivity dome. Again, the insulating behavior appears at the largest $V_{\mathrm{TG}}$, but extremely weak. In Fig. 4d, we overlaid superconducting domes with $T_{\mathrm{c}}$ defined by $0.9 R_{\mathrm{N}}, 0.5 R_{\mathrm{N}}$ and $0.1 R_{\mathrm{N}}$, respectively. Note that the superconducting dome of $\mathrm{MoS}_{2}$, which has one data point from Ref. 61 (denoted by open squares), is sketched by imitating the conductivity dome at high temperatures (Fig. S2c). Significantly, the onset $T_{\mathrm{c}}\left(0.9 R_{\mathrm{N}}\right)$ at the dome peak $\sim 5.4 \mathrm{~K}$ in $\mathrm{WSe}_{2}$ is the highest while the carrier density is the lowest among the three. According to McMillan's formula, $T_{\mathrm{c}}=\omega_{\ln } / 1.2 \exp \left[-1.04(1+\lambda) /\left(\lambda-\mu^{*}(1+0.62 \lambda)\right)\right]$ where $\lambda$ is electron-phonon coupling, $\omega_{\ln }$ is logarithmic averaged frequency and $\mu^{*}$ is Coulomb pseudopotential, the observation implies the strongest $\lambda$ in $\mathrm{WSe}_{2}$.

The evolution of superconductivity and the emergent insulator should have its correspondence in band structures. Close examination gives an important clue - the valley population with electron doping are quite distinct among the three. As shown in Fig. 4e, the energy difference between $K$ and $Q$ valleys defined as $E_{\mathrm{KQ}}$ is the smallest in $\mathrm{WSe}_{2}$, so it's easiest to occupy two valleys simultaneously with moderate gate voltages. $\mathrm{WS}_{2}$ would be more difficult and $\mathrm{MoS}_{2}$ the most difficult. This trend of multivalley population was previously identified to account for Raman softening, which is a signature of strengthened electron-phonon coupling ${ }^{62}$. Now it is again verified by the more robust superconductivity in $\mathrm{WSe}_{2}$ than $\mathrm{WS}_{2}$ and $\mathrm{MoS}_{2}$, albeit the detailed phonons involved in Raman and superconductivity may not be the same. Besides the above material engineering, multivalley effect on superconductivity could also be inferred by studying bilayer and few-layer $\mathrm{MoS}_{2}$, where increased layer number controls $E_{\mathrm{KQ}}{ }^{50,61}$. In addition to superconductivity, multivalley population may be also beneficial to charge density 
wave formation. As well as enhanced electron-phonon coupling, the $Q$ valley occupation allows for more Fermi surface nesting, both of which are essential to promote charge density wave $\mathrm{e}^{8,63-}$ ${ }^{65}$. While there are debates about the origin of CDW in TMDs, the baseline here is, multivalley population is not against the fact that one could observe a full insulating dome in $\mathrm{WSe}_{2}$ but only a remanence in $\mathrm{WS}_{2}$ and $\mathrm{MoS}_{2}$.

To quantify the above trend, we extract the Fermi energy at the superconducting dome peak (conductance peak for $\mathrm{MoS}_{2}$ ), and compare this characteristic energy with $E_{\mathrm{KQ}}$ (Fig. 4e). The same sequence among the three highlights the important role of multivalley population in both superconductivity and the emergent insulator. It's interesting to ask whether there is any possibility to observe more interesting phenomena such as correlated superconductivity and magnetism associated with the emergent insulator. With improved sample quality in the future, more investigations are certainly deserved to be conducted in various multivalley materials.

\section{Discussion}

To conclude, a detailed mapping of electronic phases has been applied to monolayer $\mathrm{WSe}_{2}$, a semiconducting TMDs dually gated by a coarse ionic liquid top gate and a fine dielectric back gate. Besides the superconducting dome with very low carrier density, we find a full insulating dome on its high-density side. The diminishing insulating phase at highest gate voltages directly excludes external mechanisms like disorder induced Anderson localization. In addition, the characterization of Hall carrier density suggests that a miniband is split from the wide conduction band. That the half filling of the miniband coincides with the insulating peak implies a Mott-like intrinsic mechanism, which matches well a $2 \sqrt{3} \times 2 \sqrt{3}$ charge density wave featuring a narrow bandwidth. To figure out the driving force, we carried out a systematic survey among the semiconducting TMDs of similar band structures, and multiple-valley population was unveiled to be a key ingredient in the evolving behavior of both superconductivity and the emergent insulator. For the former, multivalley was already shown to be able to enhance electron phonon coupling; for the latter, Fermi surface nesting from additional valleys may also result in novel charge density wave that supports strongly correlated electrons. Our result shed new light on superconductivity and emergent insulators in electron doped semiconducting 
TMDs, opening more opportunities to explore correlation physics in two dimensional van der

291 Waals materials.

\section{Methods}

\section{Fabrication of ionic liquid gated device and measurement scheme}

294

TMDCs flakes are exfoliated from bulk crystals onto silicon wafers with $285 \mathrm{~nm} \mathrm{SiO}_{2}$. Monolayer flakes identified by optical contrast are further characterized by fluorescent microscopy to select a particular part with uniform photoluminescence. It is then patterned into a standard Hall-bar geometry. Electric contacts are made by e-beam lithography followed by ebeam evaporation of $\mathrm{Ti}(1 \mathrm{~nm}) / \mathrm{Au}(50 \mathrm{~nm})$ and lift-off in hot acetone.

The ionic liquid (DEME-TFSI) is baked for half an hour before application to samples. Then the device is loaded immediately into cryostats. At temperatures above its glass transition temperature, a constant AC voltage bias $\sim 0.1 \mathrm{~V}$ is applied and source-drain current and fourprobe resistance are measured simultaneously. The DC gate voltage is then swept step by step to the maximum (sample dependent, typically $4 \sim 6 \mathrm{~V}$ ) and the leakage current is closely monitored. Then we cool down the sample at a fast rate $(\sim 3 \mathrm{~K} / \mathrm{min})$ to $180 \mathrm{~K}$ to freeze ionic liquid. Subsequently, the whole measurement process will be kept below $190 \mathrm{~K}$. To change the electronic state at low temperature, two methods are combined: one is back-gate sweeping for fine tuning, the other is thermal release of ionic liquid in a controlled way. The latter is performed around $190 \mathrm{~K}$ at which ionic liquid gating state can be released slowly. By regulating the time interval of the release, we can roughly control the ionic gating effect.

\section{Connection of resistivity mapping by back-gate transfer curves}

At each ionic gating state, the $\mathrm{SiO}_{2}$ or $\mathrm{BN}$ back gate is routinely swept and transfer curves are recorded. For the two ionic states before and after thermal release, the two transfer curves can be concatenated. In this way, each ionic state is indexed with an effective back gate voltage relative to the initial but unknown highly gated state. At the same time, Hall carrier density is measured. By extrapolating density to zero, we can get the absolute magnitude of effective gate voltage, which is denoted as $V_{\text {eff. }}$ Technical details can be found in Ref. 17. Examples for investigated samples in the main text are shown in Fig. S2. 


\section{Hall carrier density measurement}

320

Without specific notation, Hall carrier density was taken at $80 \mathrm{~K}$, at which the transfer curves were scanned in details. In Fig. S3, we plot Hall carrier density as a function of $V_{\text {eff }}$ for $\mathrm{WSe}_{2}$, $\mathrm{WS}_{2}$ and $\mathrm{MoS}_{2}$. Here the gating efficiency is quantified by an effective capacitance $C_{\text {eff }} \equiv$ $e \cdot \mathrm{d} n_{\mathrm{H}} / \mathrm{d} V_{\text {eff. }}$ The three kinds of samples share the same features: (1) at the beginning of doping, i.e. the Fermi level is close to the conduction band minima, the gating efficiency is surprisingly low with $C_{\text {eff }}=4.5 \mathrm{nF} / \mathrm{cm}^{2}$ denoted by black line. (2) Passing the low doping region, $C_{\text {eff }}$ sharply increases to the theoretical capacitance within experimental uncertainty. (3) Further doping induces an insulating behavior. Then the Hall carrier density starts to level off and even decrease with increasing $V_{\text {eff. }}$

\section{Variable Range Hopping behavior in the emergent insulator}

The temperature dependent resistivity away from the insulating peak is found to be better described by the Efros-Shklovskii variable range hopping (ES-VRH) model than the thermal activation model. The ES-VRH takes account of the coulomb interactions between the localized states on the basis of the Mott-VRH, and creates a soft gap in DOS at Fermi level. Therefore, the hopping equation in the ES-VRH model can be expressed as $R(T)=R_{0} \exp \left(\frac{T_{\mathrm{ES}}}{T}\right)^{1 / 2}$. The characteristic temperature in $2 \mathrm{D}$ limit is given by $T_{\mathrm{ES}}=\frac{2.8 e^{2}}{4 \pi \varepsilon_{r} \varepsilon_{0} k_{B} \xi_{l o c}}$, where $\varepsilon_{\mathrm{o}}$ and $\varepsilon_{\mathrm{r}}$ is the permittivity of vacuum and dielectric constant of the material. Then we can extract the localization length from the slope of $\ln R$ as function of $T^{1 / 2}$. Note that we take the dielectric constant of monolayer $\mathrm{WSe}_{2}$ from $a b$ initio calculations ${ }^{59}$.

\section{The energy difference between $K$ and $Q$ valleys}

The band structure of monolayer TMDs (i.e., $\mathrm{WSe}_{2}, \mathrm{WS}_{2}$ and $\mathrm{MoS}_{2}$ ) is well known and the DOS associated with $K$ and $Q$ valleys can be determined by their degeneracy and effective mass. So we can further calculate the chemical potential by a given electron density. However, the transport signal is not precise enough to accurately pin down the threshold electron density at which the $Q$ valley starts to be filled, hence it is difficult to determine the energy difference. As mentioned in the main text, the evolution of the superconductivity dome is closely related to the population of $K$ and $Q$ valleys. Thus we can roughly estimate $E_{\mathrm{KQ}}$ by taking the carrier density at peaks of superconducting domes for $\mathrm{WSe}_{2}$ and $\mathrm{WS}_{2}$ and the conductivity dome for 
$\mathrm{MoS}_{2}$. Particularly for $\mathrm{WSe}_{2}$, we could double check it by experimental observation of a kink in the curves of conductivity and Hall carrier density (Fig. 1b) which originate from intervalley scattering. Using this feature ${ }^{49}$, the threshold carrier density increases slightly from $3.0 \times 10^{13}$ to $\sim 3.5 \times 10^{13} \mathrm{~cm}^{-2}$, which does not affect the trend presented in Fig. 4e. The results and specific parameters we used to obtain $E_{\mathrm{KQ}}$ are listed in Supplementary Table 1.

\section{Procedures of stacking van der Waals heterostructures}

To acquire high quality samples with the surface exposed to ionic liquid, a recipe for reverted van der Waals heterostructure is followed ${ }^{66}$. We begin with spin coating polypropylene carbonate (PPC) on a silicon wafer (15 wt.\% dissolved in anisole, $3500 \mathrm{rpm}$ for $60 \mathrm{~s})$. The PPC film is manually peeled off and put onto PDMS covered with highly transparent adhesive tape. Then the stack is baked at $95^{\circ} \mathrm{C}$ for 2 minutes in order to remove bubbles and wrinkles. After cooling, the stack is mounted on a manipulator for alignment under the microscope and sequentially pick up multilayer graphene, a BN flake of 10-20 nm and monolayer TMDs. After each pick-up step, the stack is baked as before to remove contaminants or let them aggregate. Then the PPC with heterostructures on top is carefully peeled off and inverted on a final target substrate at $110^{\circ} \mathrm{C}$. At last, the whole wafer is annealed at $350^{\circ} \mathrm{C}$ for 80 mins in the vacuum of $10^{-7}$ mbar to decompose PPC without degradation of TMDCs. Details of the process are illustrated in Fig. S4.

\section{Density functional theory calculations.}

Our DFT calculations are performed with the Vienna ab initio simulation package (VASP) ${ }^{67}$. Projector augmented wave potentials ${ }^{68}$ are used with an energy cutoff of $400 \mathrm{eV}$. The generalized gradient approximation with the Perdew-Burke-Ernzerhof $f^{69}$ exchange-correlation functional is used and supplementary calculations are discussed with the plus $U$ correction of Dudarev et al. ${ }^{70}$. The $Q$-CDW state and the $M$-CDW state are modelled with a $2 \sqrt{ } 3 \times 2 \sqrt{ } 3 \times 1$ and a $2 \times 1 \times 1$ supercell, respectively. Information of the $K$-point meshes used are presented in Supplementary Table 2 . The CDW structures are generated by optimizing a manually distorted structure at each doping rate. The lateral lattice constant of the unit cell a $=3.316 \AA$, which is the optimized value at the PBE level. The CDW formation energy is defined as $E_{f}=E_{d i s}-E_{\text {undis }}$, where $E_{d i s}$ and $E_{\text {undis }}$ are the energy per unit cell of the distorted CDW structure and of the 
undistorted structure at the same doping rate, respectively. Band structures are calculated for undistorted and $Q-\mathrm{CDW}$ at various doping rates, as shown in Fig. S6 and S7, respectively. For the latter, charge distribution is also delineated in Fig. S8.

In addition, phonon dispersions are calculated with the PHONOPY interface to VASP ${ }^{71}$. The results for $\mathrm{WSe}_{2}$ is depicted in Fig. S9.

Data availability. The data that support the findings of this study are available from the corresponding author on request.

\section{References}

1. Lee, P. A., Nagaosa, N. \& Wen, X.-G. Doping a Mott insulator: Physics of high-temperature superconductivity. Rev. Mod. Phys. 78, 17-85 (2006).

2. Si, Q., Yu, R. \& Abrahams, E. High-temperature superconductivity in iron pnictides and chalcogenides. Nat. Rev. Mater. 1, 1-15 (2016).

3. Li, D. et al. Superconductivity in an infinite-layer nickelate. Nature 572, 624-627 (2019).

4. Wirth, S. \& Steglich, F. Exploring heavy fermions from macroscopic to microscopic length scales. Nat. Rev. Mater. 1, 1-16 (2016).

5. Schreiber, K. A. \& Csáthy, G. A. Competition of Pairing and Nematicity in the TwoDimensional Electron Gas. Annu. Rev. Condens. Matter Phys. 11, 17-35 (2020).

6. Cao, Y. et al. Correlated insulator behaviour at half-filling in magic-angle graphene superlattices. Nature 556, 80-84 (2018).

7. Cao, Y. et al. Unconventional superconductivity in magic-angle graphene superlattices. Nature 556, 43-50 (2018).

8. Grüner, G. The dynamics of charge-density waves. Rev. Mod. Phys. 60, 1129-1181 (1988).

9. Kim, J.-J., Yamaguchi, W., Hasegawa, T. \& Kitazawa, K. Observation of Mott Localization Gap Using Low Temperature Scanning Tunneling Spectroscopy in Commensurate 1T-TaS ${ }_{2}$. Phys. Rev. Lett. 73, 2103-2106 (1994).

10. Sipos, B. et al. From Mott state to superconductivity in 1T-TaS 2 . Nat. Mater. 7, 960-965 
(2008).

407

408

409

410

411

412

413

414

415

416

417

418

419

420

421

422

423

424

425

426

427

428

429

430

431

432

433

434

11. Yu, Y. et al. Gate-tunable phase transitions in thin flakes of $1 \mathrm{~T}-\mathrm{TaS}_{2}$. Nat. Nanotechnol. 10, 270-276 (2015).

12. Manzeli, S., Ovchinnikov, D., Pasquier, D., Yazyev, O. V. \& Kis, A. 2D transition metal dichalcogenides. Nat. Rev. Mater. 2, 1-15 (2017).

13. Mak, K. F., Xiao, D. \& Shan, J. Light-valley interactions in 2D semiconductors. Nat. Photonics 12, 451-460 (2018).

14. Wang, Q. H., Kalantar-Zadeh, K., Kis, A., Coleman, J. N. \& Strano, M. S. Electronics and optoelectronics of two-dimensional transition metal dichalcogenides. Nat. Nanotechnol. 7, 699-712 (2012).

15. Saito, Y., Nojima, T., Iwasa, Y. Highly crystalline 2D superconductors. Nat. Rev. Mater. 2 , $1-18(2016)$.

16. Ye, J. T. et al. Superconducting Dome in a Gate-Tuned Band Insulator. Science 338, 1193 $1196(2012)$.

17. Lu, J. et al. Full superconducting dome of strong Ising protection in gated monolayer $\mathrm{WS}_{2}$. Proc. Natl. Acad. Sci. 115, 3551-3556 (2018).

18. Lu, J. M. et al. Evidence for two-dimensional Ising superconductivity in gated $\mathrm{MoS}_{2}$. Science 350, 1353-1357 (2015).

19. Saito, Y. et al. Superconductivity protected by spin-valley locking in ion-gated $\mathrm{MoS}_{2}$. Nat. Phys. 12, 144-149 (2016).

20. Costanzo, D., Jo, S., Berger, H. \& Morpurgo, A. F. Gate-induced superconductivity in atomically thin $\mathrm{MoS}_{2}$ crystals. Nat. Nanotechnol. 11, 339-344 (2016).

21. Li, L. J. et al. Controlling many-body states by the electric-field effect in a two-dimensional material. Nature 529, 185-189 (2016).

22. Yao, K. et al. Optically Discriminating Carrier-Induced Quasiparticle Band Gap and Exciton Energy Renormalization in Monolayer $\mathrm{MoS}_{2}$. Phys. Rev. Lett. 119, 087401 (2017).

23. Chernikov, A. et al. Electrical Tuning of Exciton Binding Energies in Monolayer WS 2 . Phys. Rev. Lett. 115, 126802 (2015).

24. Fallahazad, B. et al. Shubnikov--de Haas Oscillations of High-Mobility Holes in 
Monolayer and Bilayer $\mathrm{WSe}_{2}$ : Landau Level Degeneracy, Effective Mass, and Negative Compressibility. Phys. Rev. Lett. 116, 086601 (2016).

25. Riley, J. M. et al. Negative electronic compressibility and tunable spin splitting in $\mathrm{WSe}_{2}$. Nat. Nanotechnol. 10, 1043-1047 (2015).

26. Larentis, S. et al. Band Offset and Negative Compressibility in Graphene-MoS 2 Heterostructures. Nano Lett. 14, 2039-2045 (2014).

27. Katoch, J. et al. Giant spin-splitting and gap renormalization driven by trions in singlelayer $\mathrm{WS}_{2} / \mathrm{h}-\mathrm{BN}$ heterostructures. Nat. Phys. 14, 355 (2018).

28. Ovchinnikov, D. et al. Disorder engineering and conductivity dome in $\mathrm{ReS}_{2}$ with electrolyte gating. Nat. Commun. 7, 1-7 (2016).

29. Nelson, J. \& Goldman, A. M. Metallic state of low-mobility silicon at high carrier density induced by an ionic liquid. Phys. Rev. B 91, 241304 (2015).

30. Allain, A. \& Kis, A. Electron and Hole Mobilities in Single-Layer WSe 2 . ACS Nano 8, $7180-7185$ (2014).

31. Saito, Y., Kasahara, Y., Ye, J., Iwasa, Y. \& Nojima, T. Metallic ground state in an ion-gated two-dimensional superconductor. Science 350, 409-413 (2015).

32. Xia, Y., Xie, W., Ruden, P. P. \& Frisbie, C. D. Carrier Localization on Surfaces of Organic Semiconductors Gated with Electrolytes. Phys. Rev. Lett. 105, 036802 (2010).

33. Xie, W., Liu, F., Shi, S., Ruden, P. P. \& Frisbie, C. D. Charge Density Dependent TwoChannel Conduction in Organic Electric Double Layer Transistors (EDLTs). Adv. Mater. 26, 2527-2532 (2014).

34. Xie, W., Wang, S., Zhang, X., Leighton, C. \& Frisbie, C. D. High Conductance 2D Transport around the Hall Mobility Peak in Electrolyte-Gated Rubrene Crystals. Phys. Rev. Lett. 113, 246602 (2014).

35. Xie, W. \& Frisbie, C. D. Organic Electrical Double Layer Transistors Based on Rubrene Single Crystals: Examining Transport at High Surface Charge Densities above $10^{13} \mathrm{~cm}^{-2}$. J. Phys. Chem. C 115, 14360-14368 (2011).

36. Wang, F. et al. Ionic Liquid Gating of Suspended $\mathrm{MoS}_{2}$ Field Effect Transistor Devices. Nano Lett. 15, 5284-5288 (2015). 
37. Petach, T. A. et al. Disorder from the Bulk Ionic Liquid in Electric Double Layer Transistors. ACS Nano 11, 8395-8400 (2017).

38. Browning, A. et al. Evaluation of disorder introduced by electrolyte gating through transport measurements in graphene. Appl. Phys. Express 9, 065102 (2016).

39. Rösner, M., Haas, S. \& Wehling, T. O. Phase diagram of electron-doped dichalcogenides. Phys. Rev. B 90, 245105 (2014).

40. Liu, G.-B., Xiao, D., Yao, Y., Xu, X. \& Yao, W. Electronic structures and theoretical modelling of two-dimensional group-VIB transition metal dichalcogenides. Chem. Soc. Rev. 44, 2643-2663 (2015).

41. Movva, H. C. P. et al. Density-Dependent Quantum Hall States and Zeeman Splitting in Monolayer and Bilayer WSe 2 . Phys. Rev. Lett. 118, 247701 (2017).

42. Shi, Q. et al. Odd- and even-denominator fractional quantum Hall states in monolayer $\mathrm{WSe}_{2}$. Nat. Nanotechnol. 15, 569-573 (2020).

43. Kormányos, A. et al. k.p theory for two-dimensional transition metal dichalcogenide semiconductors. 2D Mater. 2, 022001 (2015).

44. Liu, G.-B., Shan, W.-Y., Yao, Y., Yao, W. \& Xiao, D. Three-band tight-binding model for monolayers of group-VIB transition metal dichalcogenides. Phys. Rev. B 88, 085433 (2013).

45. Nguyen, P. V. et al. Visualizing electrostatic gating effects in two-dimensional heterostructures. Nature 572, 220-223 (2019).

46. Zhang, C. et al. Probing Critical Point Energies of Transition Metal Dichalcogenides: Surprising Indirect Gap of Single Layer WSe 2 . Nano Lett. 15, 6494-6500 (2015).

47. Hsu, W.-T. et al. Evidence of indirect gap in monolayer WSe 2 . Nat. Commun. 8, 1-7 (2017).

48. Ye, J. et al. Accessing the transport properties of graphene and its multilayers at high carrier density. Proc. Natl. Acad. Sci. 108, 13002-13006 (2011).

49. Zhang, H., Berthod, C., Berger, H., Giamarchi, T. \& Morpurgo, A. F. Band Filling and Cross Quantum Capacitance in Ion-Gated Semiconducting Transition Metal Dichalcogenide Monolayers. Nano Lett. 19, 8836-8845 (2019).

50. Piatti, E. et al. Multi-Valley Superconductivity in Ion-Gated MoS 2 Layers. Nano Lett. 18, 

4821-4830 (2018).

51. Brumme, T., Calandra, M. \& Mauri, F. Determination of scattering time and of valley occupation in transition-metal dichalcogenides doped by field effect. Phys. Rev. B 93, 081407 (2016).

52. Dean, C. R. et al. Boron nitride substrates for high-quality graphene electronics. Nat. Nanotechnol. 5, 722-726 (2010).

53. Geim, A. K. \& Grigorieva, I. V. Van der Waals heterostructures. Nature 499, 419-425 (2013).

54. Efros, A. L. \& Shklovskii, B. I. Coulomb gap and low temperature conductivity of disordered systems. J. Phys. C Solid State Phys. 8, L49-L51 (1975).

55. Perfetti, L. et al. Spectroscopic Signatures of a Bandwidth-Controlled Mott Transition at the Surface of 1T-TaSe 2 . Phys. Rev. Lett. 90, 166401 (2003).

56. Colonna, S. et al. Mott Phase at the Surface of $1 \mathrm{~T}-\mathrm{TaSe}_{2}$ Observed by Scanning Tunneling Microscopy. Phys. Rev. Lett. 94, 036405 (2005).

57. Nakata, Y. et al. Monolayer $1 \mathrm{~T}-\mathrm{NbSe}_{2}$ as a Mott insulator. NPG Asia Mater. 8, e321-e321 (2016).

58. Monarkha, Yu. P. \& Syvokon, V. E. A two-dimensional Wigner crystal. Low Temp. Phys. 38, 1067-1095 (2012).

59. Laturia, A., Put, M. L. V. de \& Vandenberghe, W. G. Dielectric properties of hexagonal boron nitride and transition metal dichalcogenides: from monolayer to bulk. Npj 2D Mater. Appl. 2, 1-7 (2018).

60. Giuliani, G. \& Vignale, G. Quantum Theory of the Electron Liquid. (Cambridge University Press, 2005).

61. Fu, Y. et al. Gated tuned superconductivity and phonon softening in monolayer and bilayer MoS 2. Npj Quantum Mater. 2, 1-7 (2017).

62. Sohier, T. et al. Enhanced Electron-Phonon Interaction in Multivalley Materials. Phys. Rev. $X \mathbf{9}, 031019$ (2019).

63. Withers, R. L. \& Wilson, J. A. An examination of the formation and characteristics of charge-density waves in inorganic materials with special reference to the two- and one- 
dimensional transition-metal chalcogenides. J. Phys. C Solid State Phys. 19, 4809-4845 (1986).

64. Castro Neto, A. H. Charge Density Wave, Superconductivity, and Anomalous Metallic Behavior in 2D Transition Metal Dichalcogenides. Phys. Rev. Lett. 86, 4382-4385 (2001).

65. Wilson, J. A., Di Salvo, F. J. \& Mahajan, S. Charge-Density Waves in Metallic, Layered, Transition-Metal Dichalcogenides. Phys. Rev. Lett. 32, 882-885 (1974).

66. Li, X.-X. et al. Gate-controlled reversible rectifying behaviour in tunnel contacted atomically-thin $\mathrm{MoS}_{2}$ transistor. Nat. Commun. 8, 1-7 (2017).

67. Kresse, G. \& Furthmüller, J. Efficient iterative schemes for ab initio total-energy calculations using a plane-wave basis set. Phys. Rev. B 54, 11169-11186 (1996).

68. Kresse, G. \& Joubert, D. From ultrasoft pseudopotentials to the projector augmented-wave method. Phys. Rev. B 59, 1758-1775 (1999).

69. Perdew, J. P., Burke, K. \& Ernzerhof, M. Generalized Gradient Approximation Made Simple. Phys. Rev. Lett. 77, 3865-3868 (1996).

70. Dudarev, S. L., Botton, G. A., Savrasov, S. Y., Humphreys, C. J. \& Sutton, A. P. Electronenergy-loss spectra and the structural stability of nickel oxide: An LSDA+U study. Phys. Rev. B 57, 1505-1509 (1998).

71. Togo, A. \& Tanaka, I. First principles phonon calculations in materials science. Scr. Mater. 108, 1-5 (2015).

\section{Acknowledgements}

J.M.L. acknowledges supports from NSFC No. 11974027, National Key R\&D Program of China No. 2019YFA0307800 and Beijing Natural Science Foundation No. Z190011. C.J. is supported by NSFC No. 11974024, National Key R\&D Program of China No. 2016YFA030091 and the Strategic Priority Research Program of the Chinese Academy of Sciences under grant number XDB33000000. Y.H. is supported by National Key R\&D Program of China No. 2019YFA0308000, NSFC 11874405 and the Youth Innovation Promotion Association of CAS (2019007). Z.M.L. is supported by NSFC 61825401 and National Key R\&D Program of China No. 2018YFA0703703. 
a

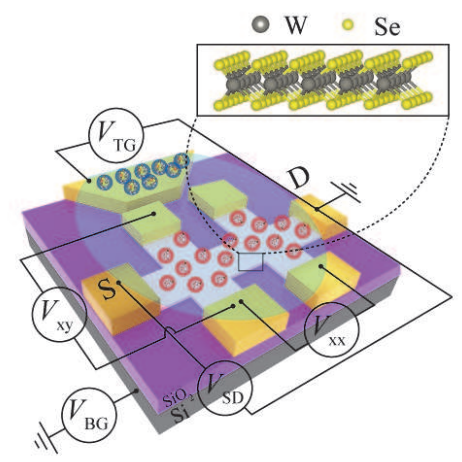

b

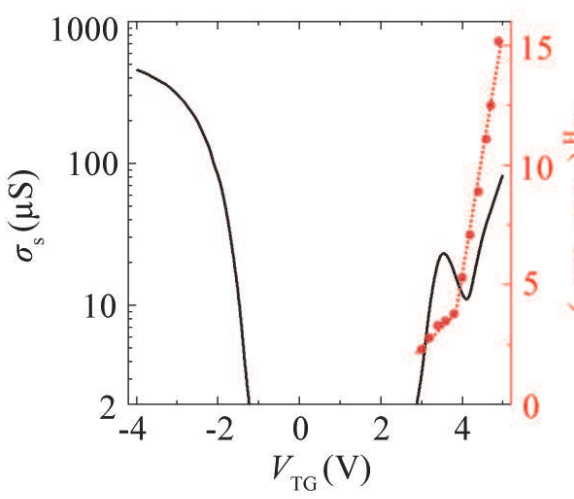

c

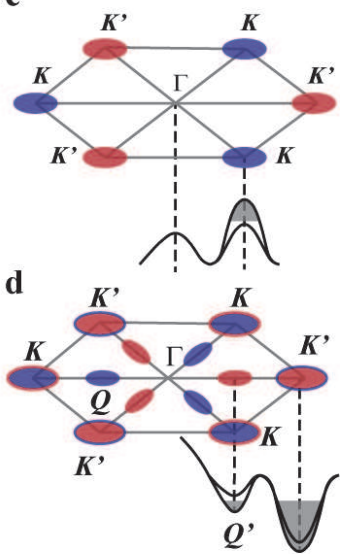

e

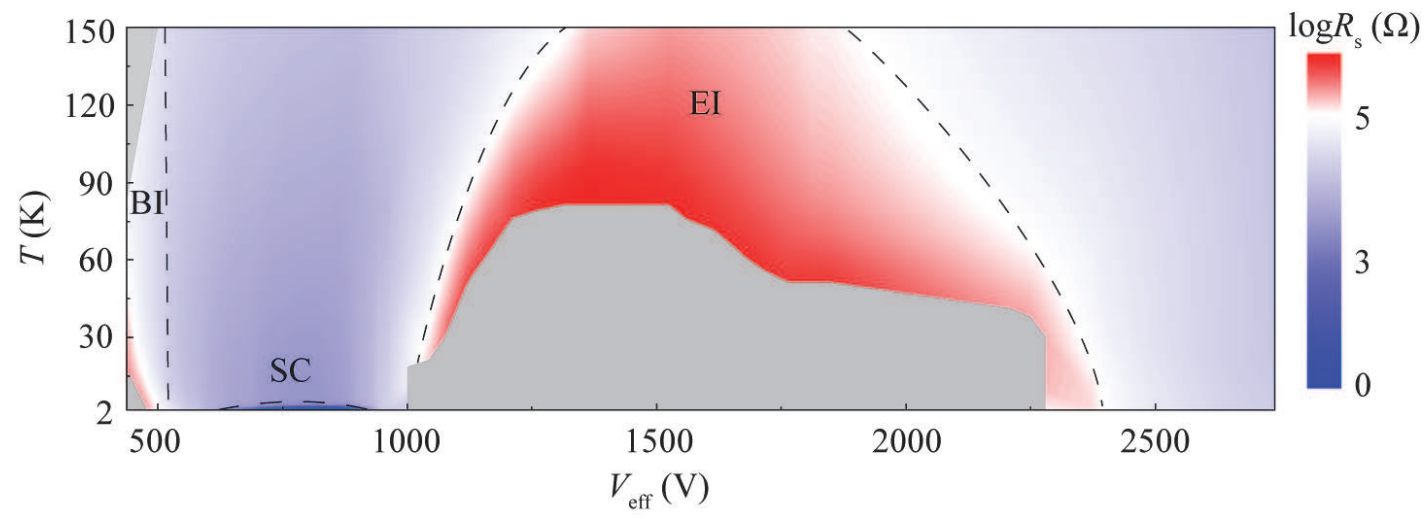

551

552

553

554

555

556

557

558

559

560

561

562

563

564

565

Figure 1 Observation of an emergent insulator in ionic gated monolayer $\mathrm{WSe}_{2}$. a.

Schematics of an ionic liquid field effect transistor made of monolayer $\mathrm{WSe}_{2}$. The top gate $\left(V_{\mathrm{TG}}\right)$ is for ionic liquid and the back gate $\left(V_{\mathrm{BG}}\right)$ is for $285 \mathrm{~nm} \mathrm{SiO}_{2}$ dielectric capacitor. b. Conductivity and Hall carrier density plotted to the left and right axes, respectively, as a function of ionic liquid gate voltages. While the conductivity tends to saturate in the hole regime, there is a significant dip on the electron side that is typically attributed to the intervalley scattering between $K$ and $Q$ valleys (shown in d). c-d. Valley filling for hole (c) and electron (d) doping. In the hole doped regime, only $K / K^{\prime}$ pockets at the corners of the hexagonal Brillouin zone are occupied, whereas in the electron doped regime both $K$ and $Q$ valleys may be populated. Red and blue colors indicate up and down spins, respectively. e. Resistivity mapping as a function of temperature and ionic liquid gating, showing a series of electronic phases from a band insulator (BI), superconductivity (SC), an emergent insulator (EI) to a metal. Note that here the ionic liquid gating effect has been converted to the effective back gate $V_{\text {eff. }}$ 
a

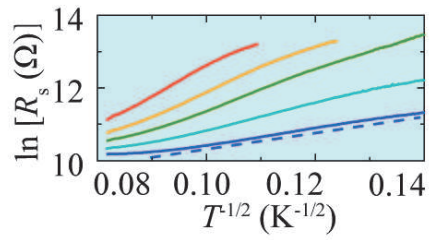

b

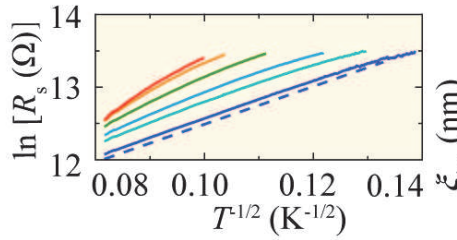

c

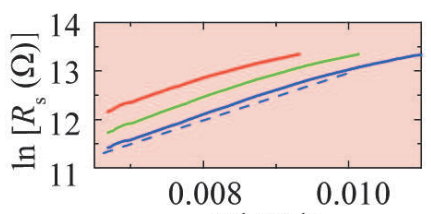

e

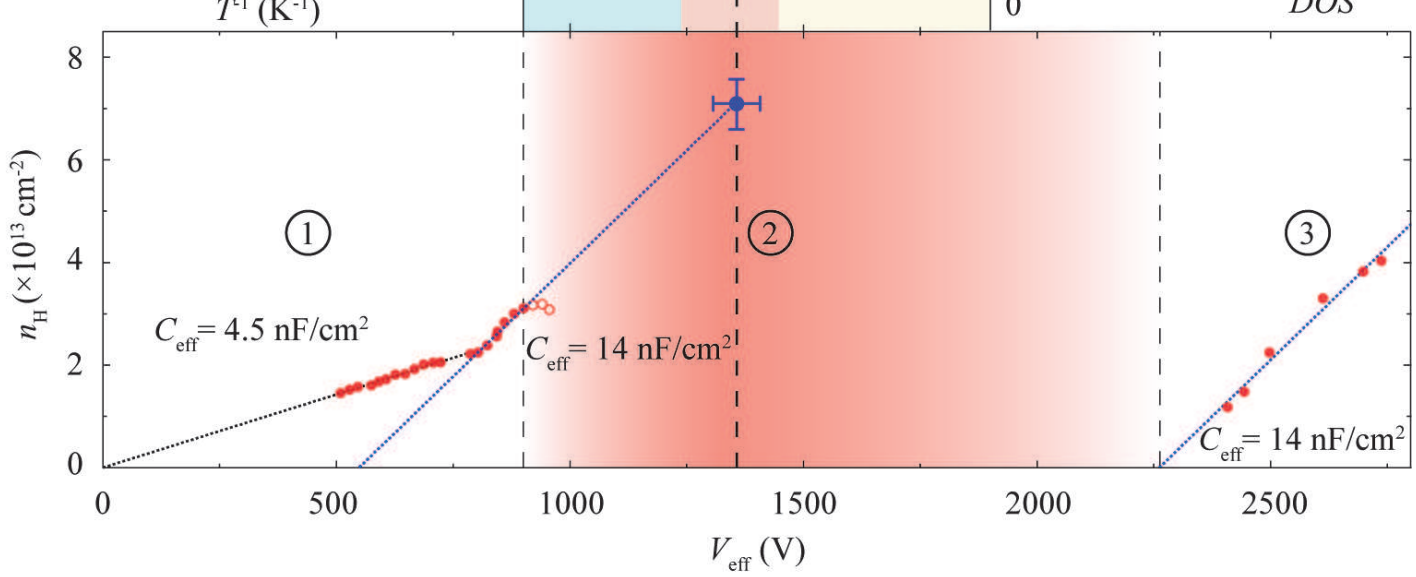

d
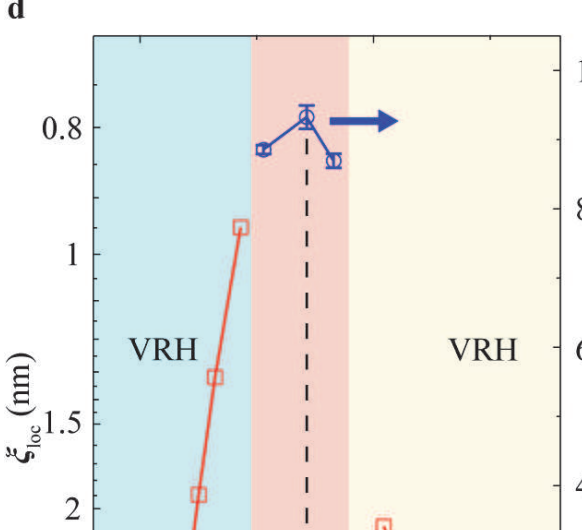

00

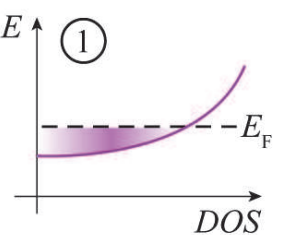

80
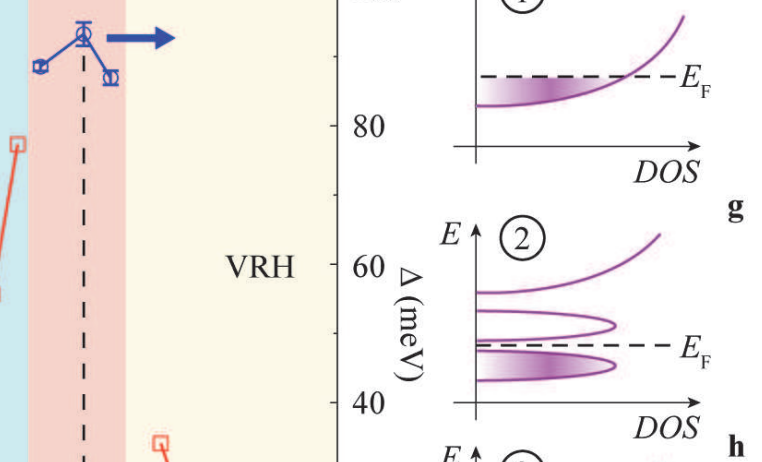

VRH
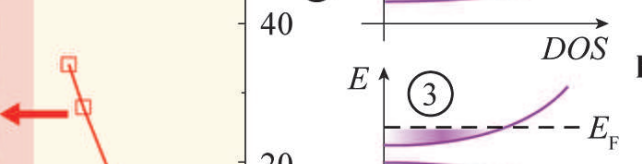

20

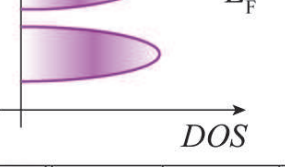

566

Figure 2 Identification of a narrow band corresponding to the insulator. a-c.

Characterization of temperature dependent resistivity across the dome-like insulating phase. In the low- (a) and high (b) density regimes, they are well fitted by Efros-Shklovskii variablerange hopping, while the most insulating peak (c) could be fitted by a thermal activation model. d. The derived localization length and energy gap are plotted to the left and right axes, respectively. e. The Hall carrier density map is divided into three regions, the middle of which is highlighted by red colors for the insulating phase. The black dotted line in region 1 has a slope of $C_{\text {eff }}=4.5 \mathrm{nF} / \mathrm{cm}^{2}$, which is only $1 / 3$ of the expected capacitance. With higher gate voltages, the slope indicated by the blue dotted line restores to be normal except those close to the insulator (open symbols). The extrapolated carrier density for the insulating peak is approximately $7.3 \times 10^{13} \mathrm{~cm}^{-2}$, in excellent agreement with the measured carrier density at high temperatures $\sim 7.1 \times 10^{13} \mathrm{~cm}^{-2}$ (solid circles in blue). The intercept in region 3 corresponds to a density of injected electrons $\sim 1.5 \times 10^{14} \mathrm{~cm}^{-2}$ (at $V_{\text {eff }} \sim 2260 \mathrm{~V}$ ), which almost doubles that of the insulating peak. $\mathbf{f}-\mathbf{h}$. Schematics of band fillings for three regimes indexed in $\mathbf{e}$ including superconductivity (f), the emerging insulator (g) and the right metal (h). 
a

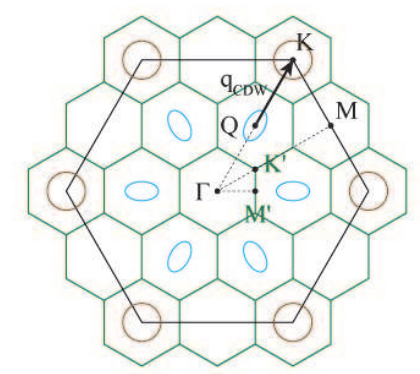

c

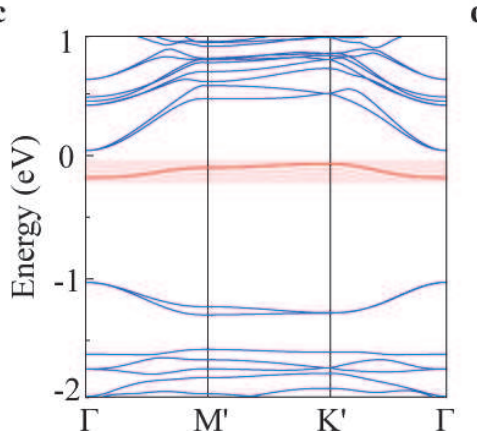

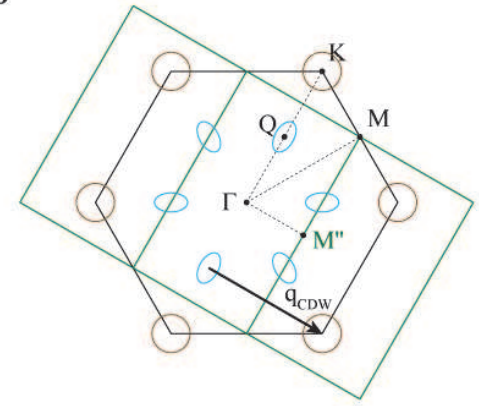

d

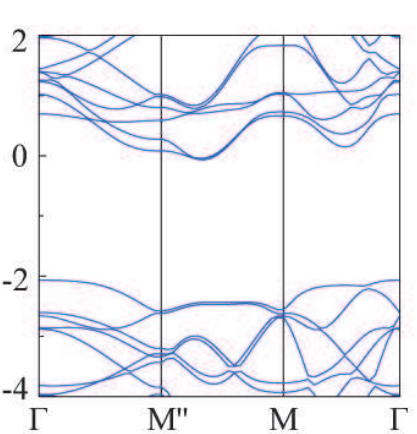

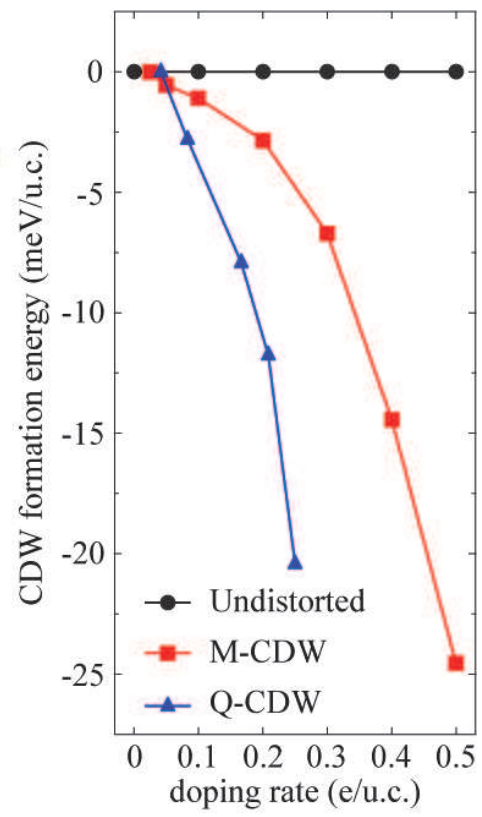

583

584

585

586

587

588

589

590

Figure 3 Charge density wave with a narrow band. a-b. Reconstructed (green) and original (black) Brillouin zones for the $2 \sqrt{3} \times 2 \sqrt{3} Q$-CDW (a) and the $2 \times 1 M$-CDW (b), where unreconstructed Fermi surfaces (circles and ellipses) and CDW wavevectors (arrows) are indicated. c-d. Corresponding band dispersions along high-symmetry directions. In c, the narrow band is highlighted in red. e. Formation energy of the $M$-CDW and $Q-\mathrm{CDW}$ states as a function of electron doping rates in DFT calculations. 


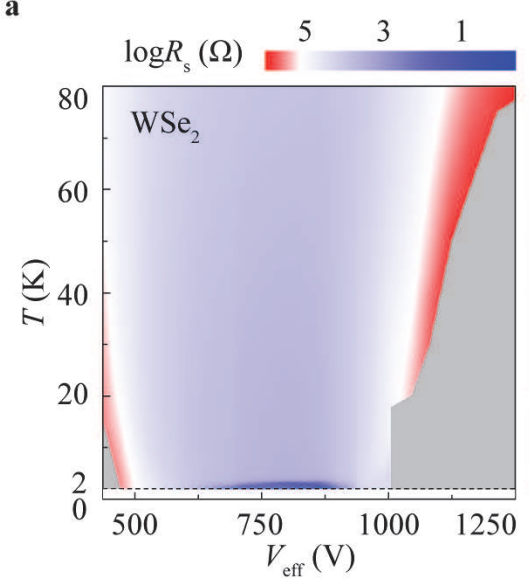

d

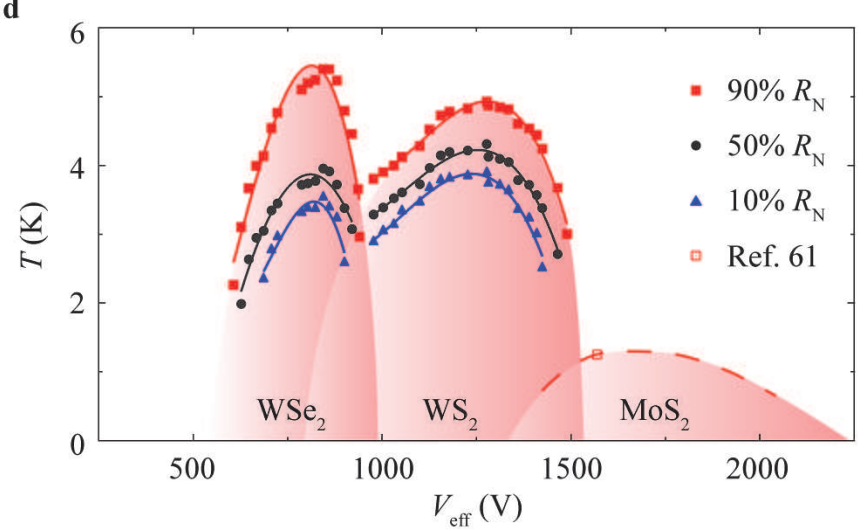

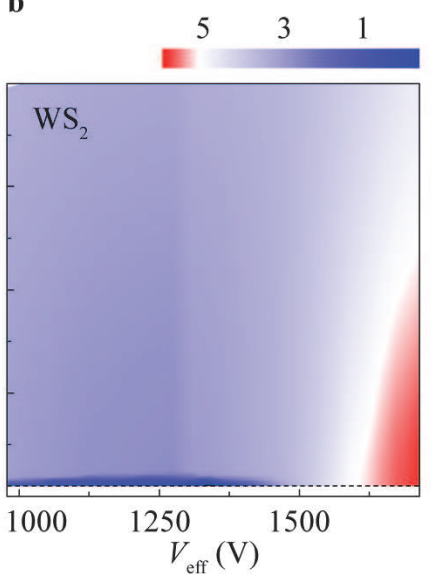
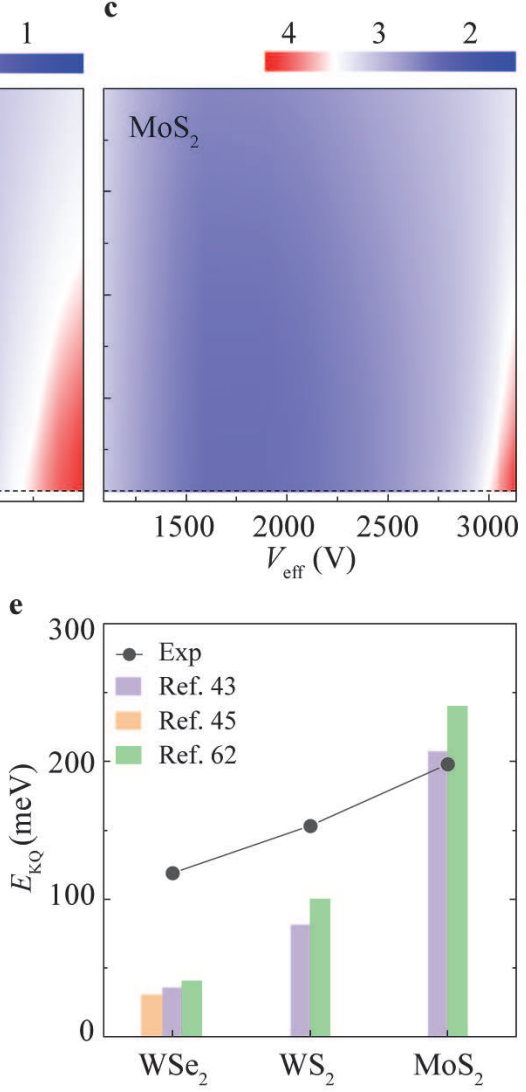

591

Figure 4 Evolution of the emergent insulator and superconductivity across the

593

semiconducting class of TMDs. a-c. Resistivity mapping as a function of temperature and gating effect for monolayer $\mathrm{WSe}_{2}(\mathbf{a}), \mathrm{WS}_{2}(\mathbf{b})$ and $\mathrm{MoS}_{2}(\mathbf{c})$, showing the similarity between the three and gradual and systematic changes due to evolving band structures. d. Summarized superconducting domes. For monolayer $\mathrm{MoS}_{2}$, no superconductivity was observed above $2 \mathrm{~K}$ in the present experiment. The open square is taken from Ref. 61 and the dome (dashed line) mimics the conductivity dome at high temperatures. e. The filled bars represent the energy difference between $K$ and $Q$ valleys obtained by both theories and experiments in the literature. Extracted values from dome peaks roughly follow the trend (see Methods, section 5). 
a

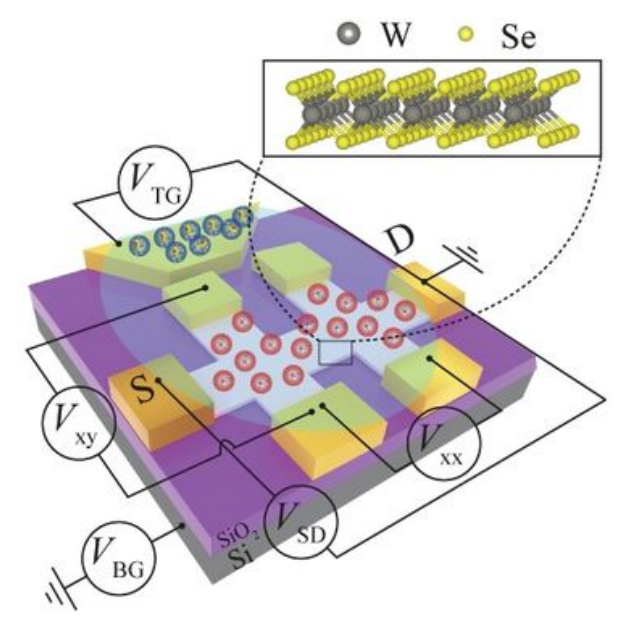

b

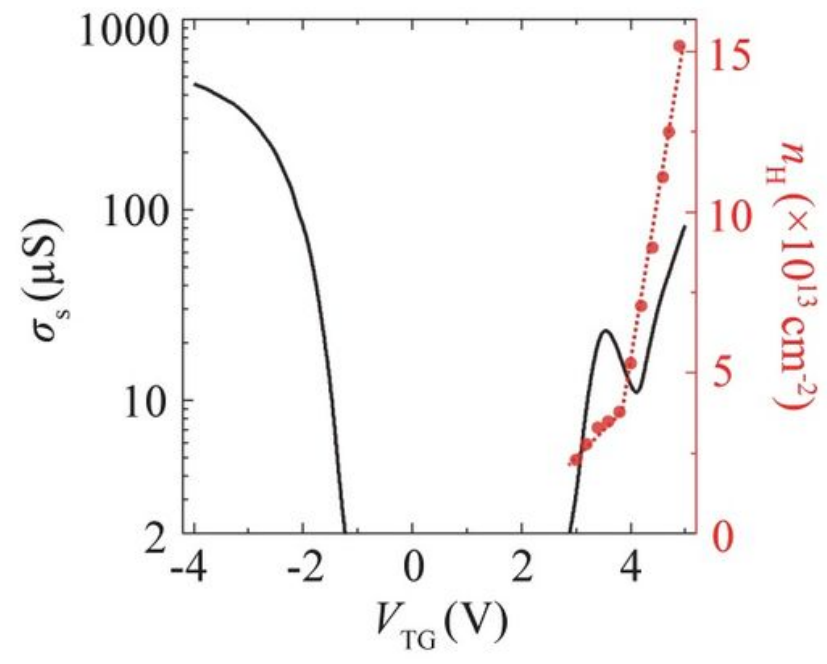

c

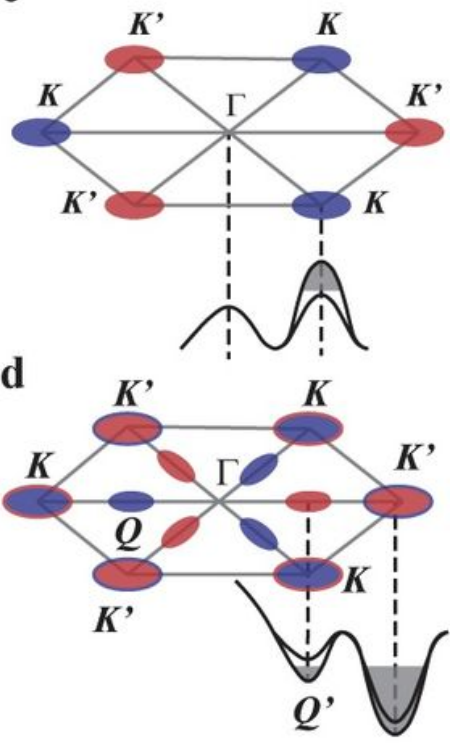

e

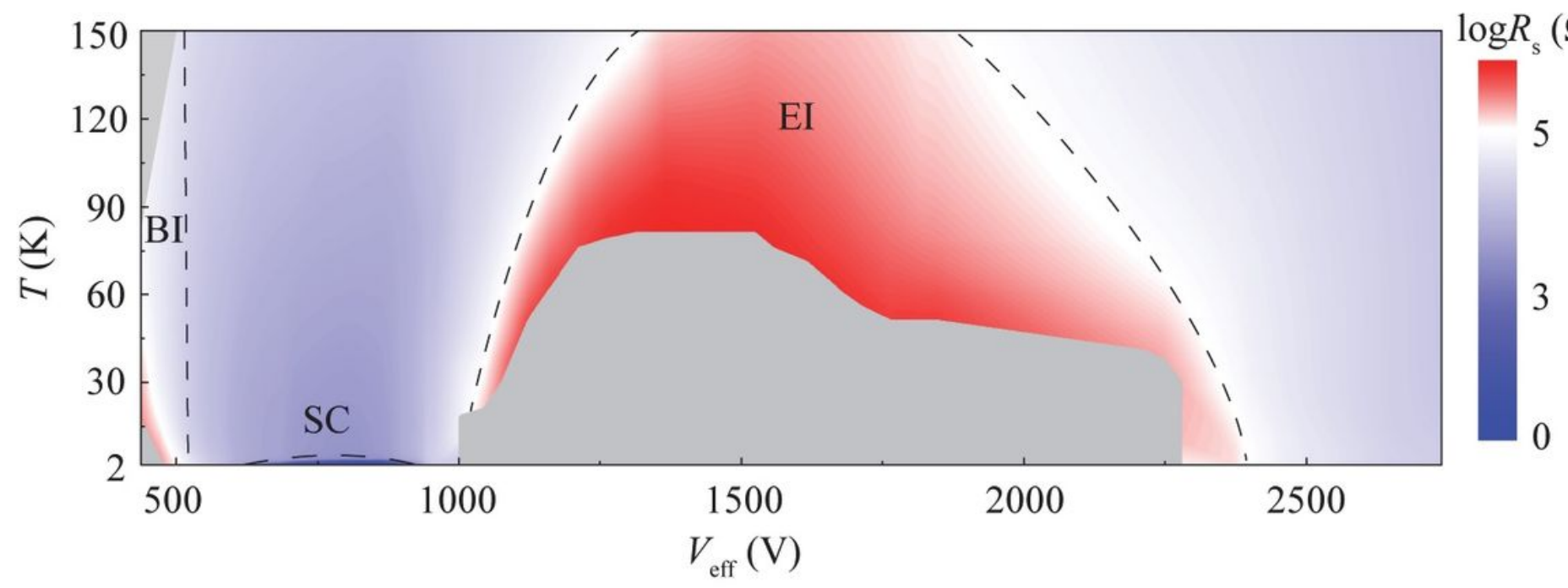

\section{Figure 1}

Observation of an emergent insulator in ionic gated monolayer WSe2. a.Schematics of an ionic liquid field effect transistor made of monolayer WSe2. The top gate (VTG) is for ionic liquid and the back gate (VBG) is for $285 \mathrm{~nm} \mathrm{SiO2} \mathrm{dielectric} \mathrm{capacitor.} \mathrm{b.} \mathrm{Conductivity} \mathrm{and} \mathrm{Hall} \mathrm{carrier} \mathrm{density} \mathrm{plotted} \mathrm{to} \mathrm{the} \mathrm{left}$ and right axes, respectively, as a function of ionic liquid gate voltages. While the conductivity tends to saturate in the hole regime, there is a significant dip on the electron side that is typically attributed to the intervalley scattering between $K$ and Q valleys (shown in d). c-d. Valley filling for hole (c) and electron (d) doping. In the hole doped regime, only K/K' pockets at the corners of the hexagonal Brillouin zone are occupied, whereas in the electron doped regime both $\mathrm{K}$ and $\mathrm{Q}$ valleys may be populated. Red and blue colors indicate up and down spins, respectively. e. Resistivity mapping as a function of temperature and ionic liquid gating, showing a series of electronic phases from a band insulator (BI), superconductivity 
(SC), an emergent insulator (EI) to a metal. Note that here the ionic liquid gating effect has been converted to the effective back gate Veff.

a

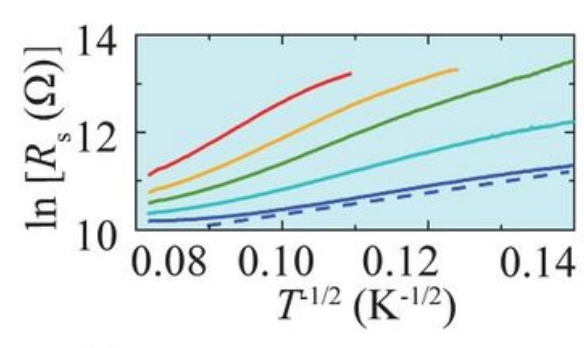

b

c
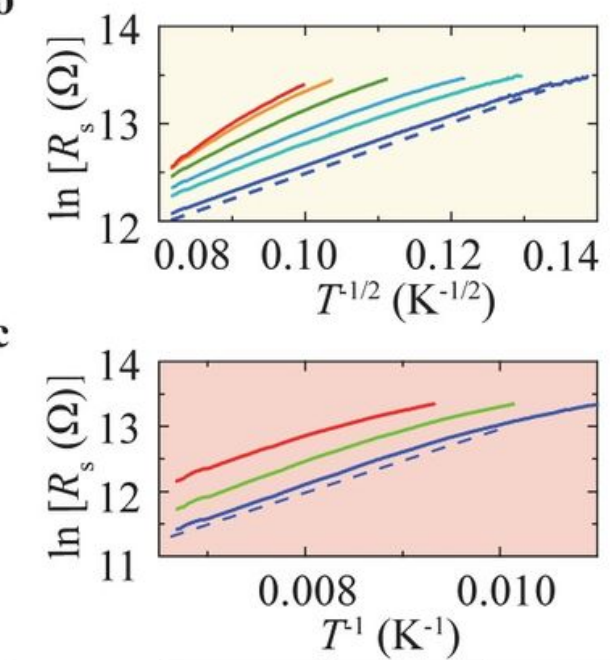

e

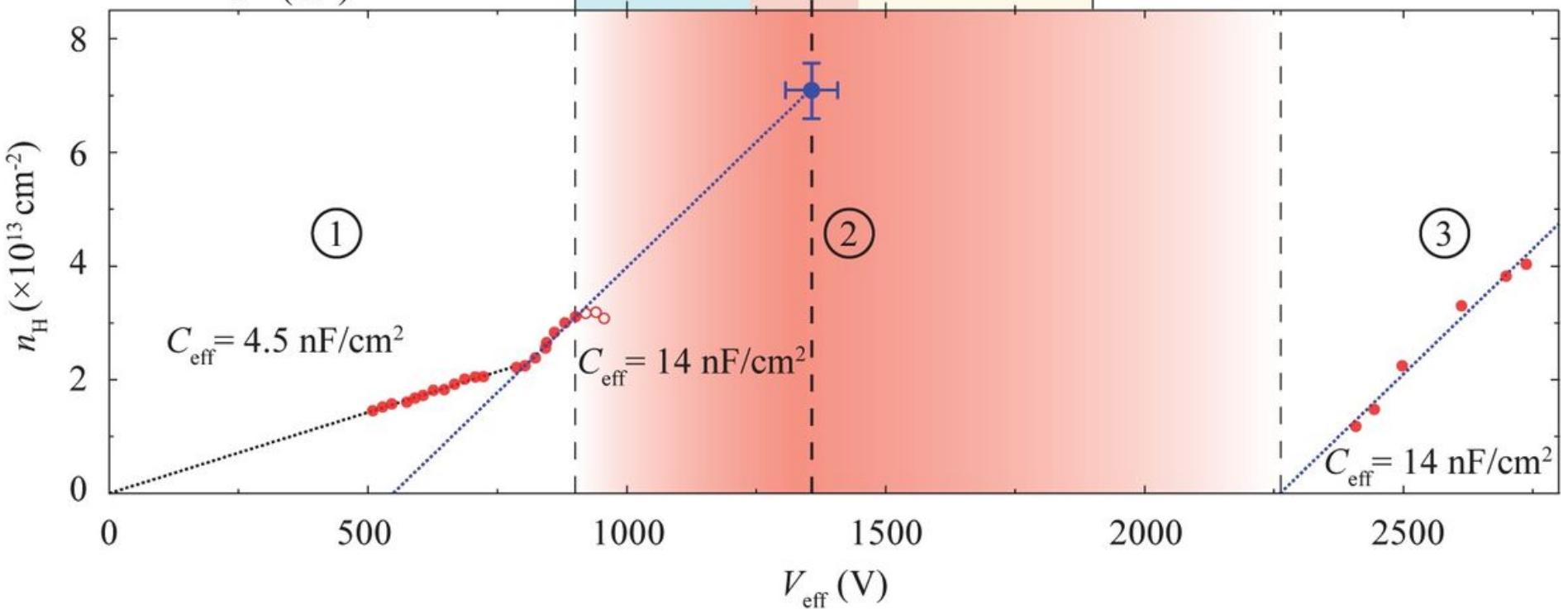

d
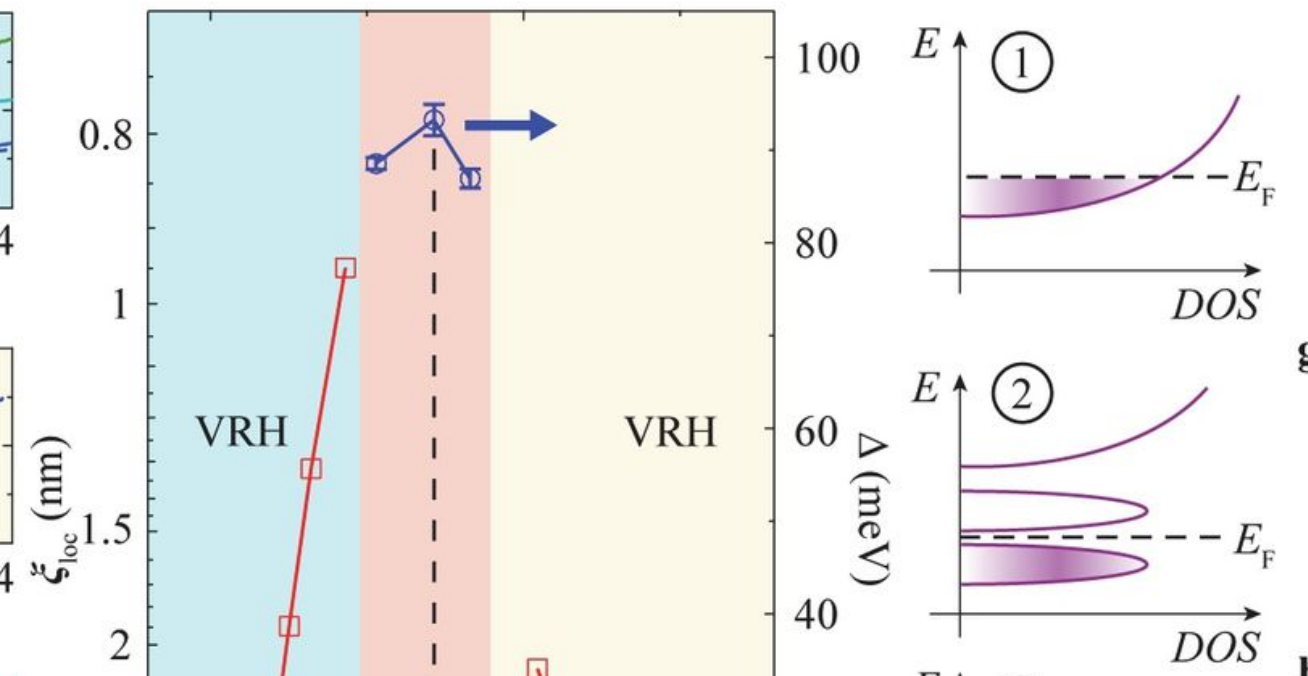

\section{Figure 2}

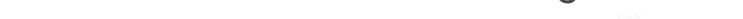


slope indicated by the blue dotted line restores to be normal except those close to the insulator (open symbols). The extrapolated carrier density for the insulating peak is approximately $7.3 \times 1013 \mathrm{~cm}-2$, in excellent agreement with the measured carrier density at high temperatures $\sim 7.1 \times 1013 \mathrm{~cm}-2$ (solid circles in blue). The intercept in region 3 corresponds to a density of injected electrons $~ 1.5 \times 1014 \mathrm{~cm}-2$ (at Veff $\sim 2260 \mathrm{~V}$ ), which almost doubles that of the insulating peak. $\mathrm{f}$-h. Schematics of band fillings for three regimes indexed in e including superconductivity $(\mathrm{f})$, the emerging insulator $(\mathrm{g})$ and the right metal $(\mathrm{h})$.

a

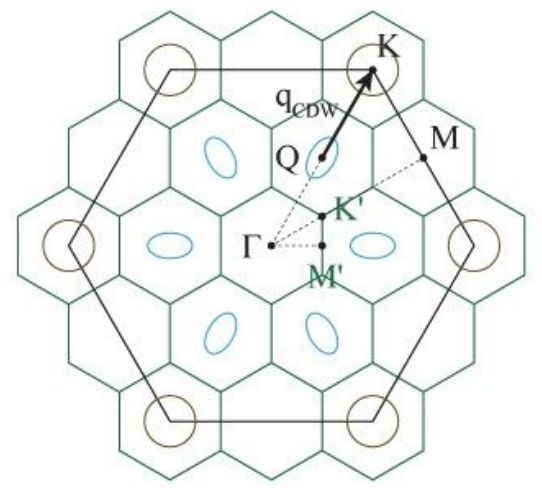

c

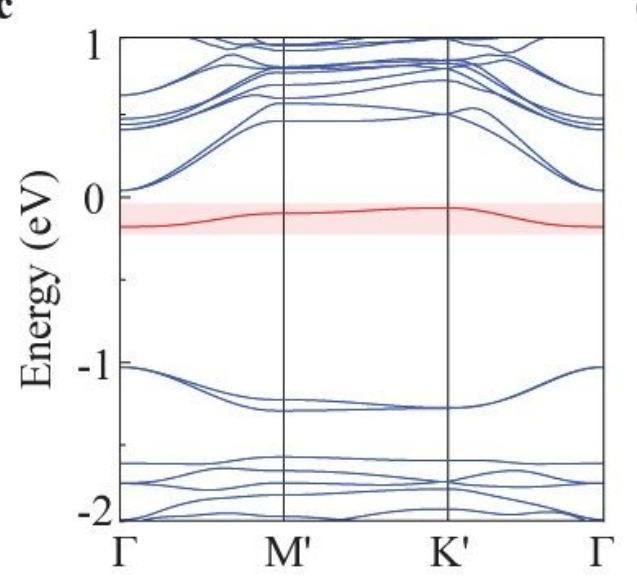

b

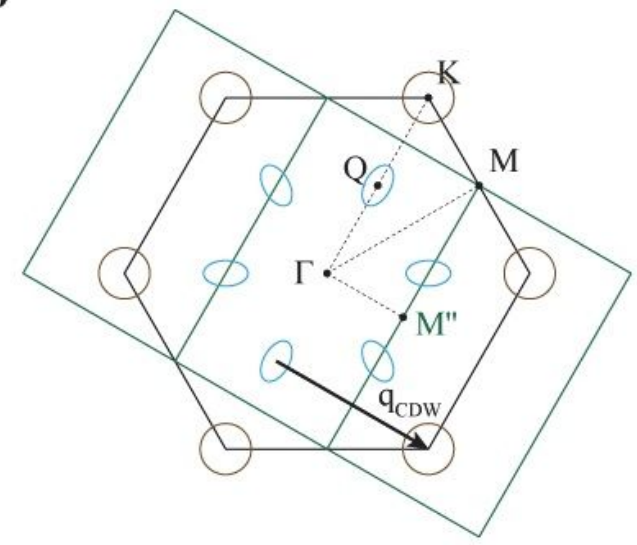

d

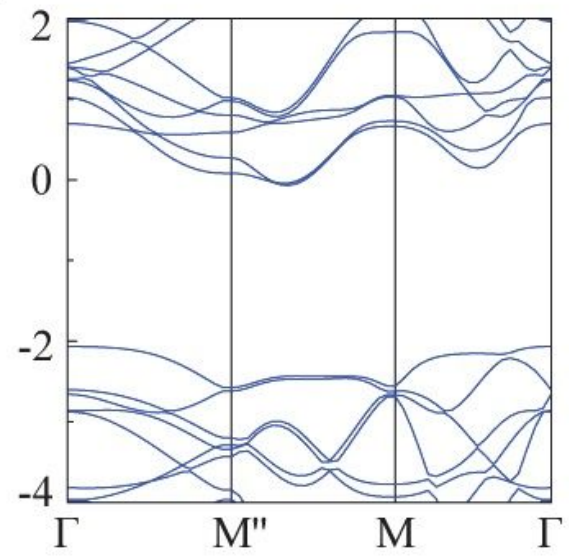

e

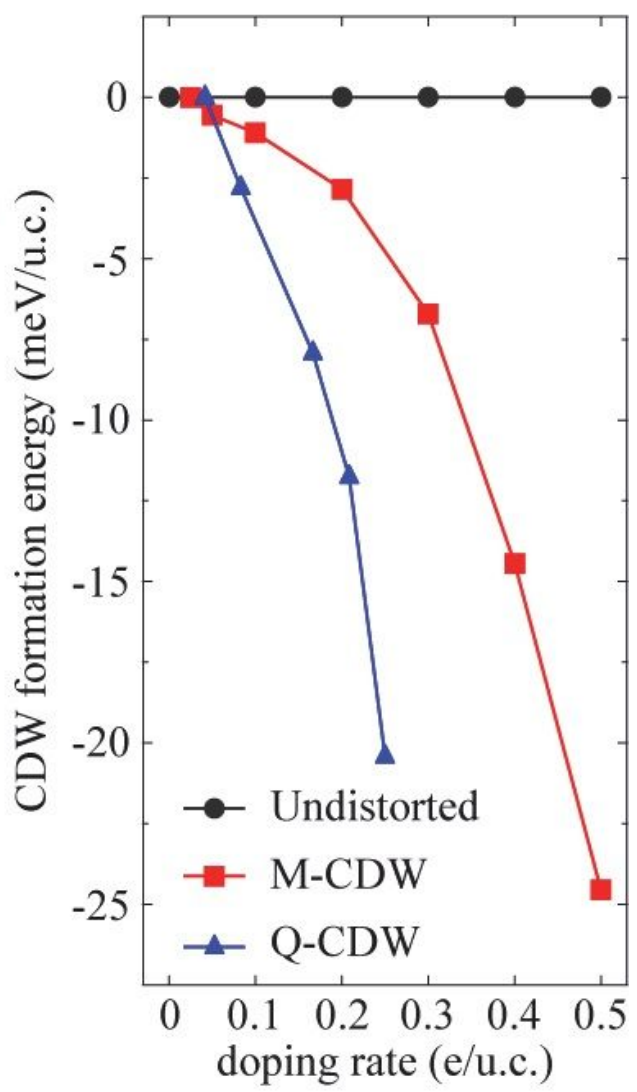

\section{Figure 3}

Charge density wave with a narrow band. a-b. Reconstructed (green) and original (black) Brillouin zones for the $2 \sqrt{ } 3 \times 2 \sqrt{ } 3 \mathrm{Q}-\mathrm{CDW}(\mathrm{a})$ and the $2 \times 1 \mathrm{M}-\mathrm{CDW}(\mathrm{b})$, where unreconstructed Fermi surfaces (circles and ellipses) and CDW wavevectors (arrows) are indicated. c-d. Corresponding band dispersions along highsymmetry directions. In c, the narrow band is highlighted in red. e. Formation energy of the M-CDW and QCDW states as a function of electron doping rates in DFT calculations. 
a

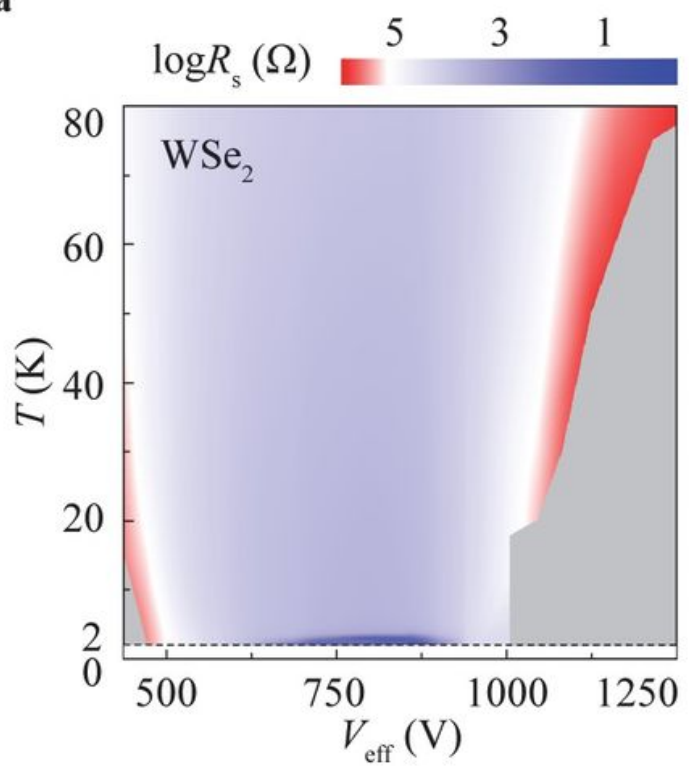

d

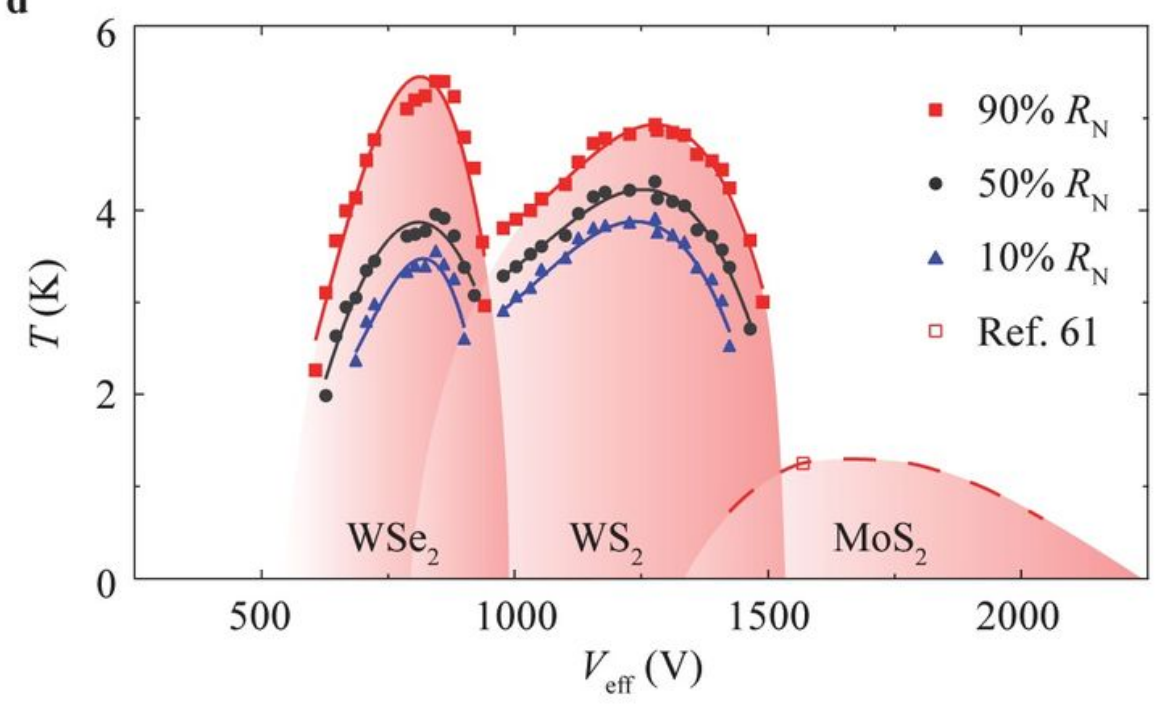

b

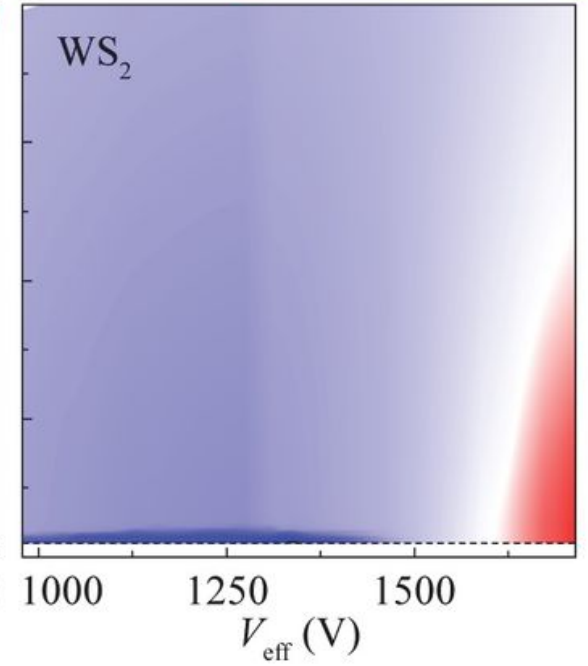

c

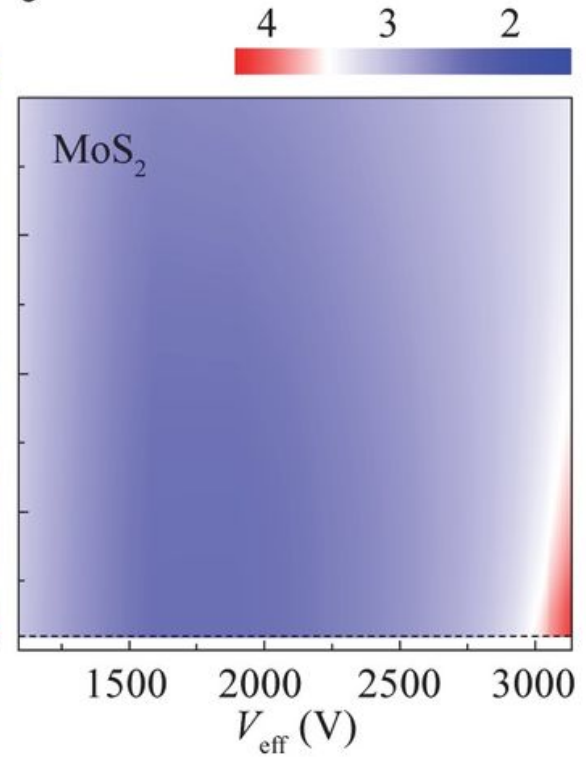

e

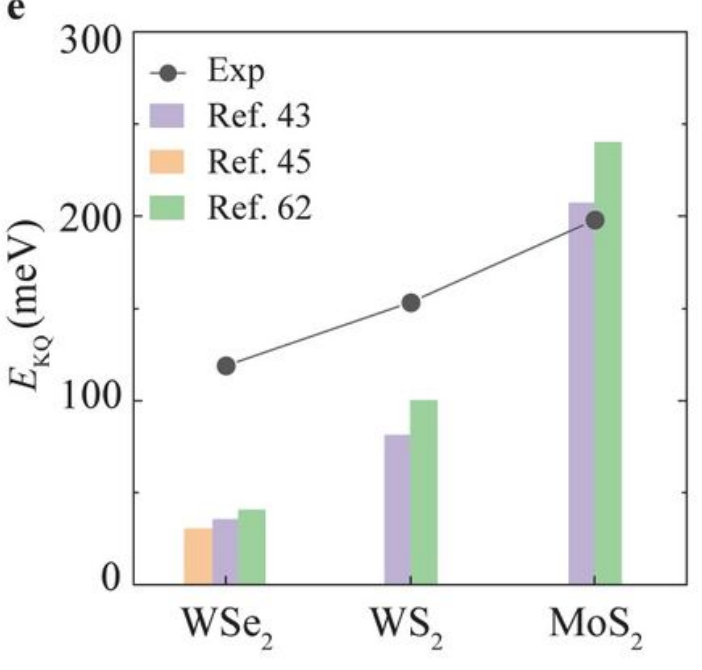

\section{Figure 4}

Evolution of the emergent insulator and superconductivity across the semiconducting class of TMDs. a-c. Resistivity mapping as a function of temperature and gating effect for monolayer WSe2 (a), WS2 (b) and MoS2 (c), showing the similarity between the three and gradual and systematic changes due to evolving band structures. $d$. Summarized superconducting domes. For monolayer MoS2, no superconductivity was observed above $2 \mathrm{~K}$ in the present experiment. The open square is taken from Ref. 61 and the dome (dashed line) mimics the conductivity dome at high temperatures. e. The filled bars represent the energy difference between $\mathrm{K}$ and $\mathrm{Q}$ valleys obtained by both theories and experiments in the literature. Extracted values from dome peaks roughly follow the trend (see Methods, section 5).

\section{Supplementary Files}

This is a list of supplementary files associated with this preprint. Click to download. 
- Manuscriptsupplementaryinformation.docx 